\title{
Concurrent Goal-Based Execution of Constraint Handling Rules
}

\author{
Edmund S. L. Lam \\ School of Computing, National University of Singapore \\ S16 Level 5, 3 Science Drive 2, Singapore 117543 \\ lamsoonl@comp.nus.edu.sg \\ Martin Sulzmann \\ Informatik Consulting Systems AG \\ martin.sulzmann@gmail.com \\ submitted 5 December 2008; revised 18 January 2010; accepted 31 May 2010
}

\begin{abstract}
We introduce a systematic, concurrent execution scheme for Constraint Handling Rules (CHR) based on a previously proposed sequential goal-based CHR semantics. We establish strong correspondence results to the abstract CHR semantics, thus guaranteeing that any answer in the concurrent, goal-based CHR semantics is reproducible in the abstract CHR semantics. Our work provides the foundation to obtain efficient, parallel CHR execution schemes.
\end{abstract}

KEYWORDS: multi-set rewriting, constraints, concurrency

\section{Introduction}

Rewriting is a powerful discipline to specify the semantics of programming languages and to perform automated deduction. There are numerous flavors of rewriting such as term, graph rewriting etc. Our focus here is on exhaustive, forward chaining, multi-set constraint rewriting as found in Constraint Handling Rules (CHR) (Frühwirth 1998) which are used in a multitude of applications such as general purpose constraint programming, type system design, agent specification and planning etc (Frühwirth 2006). Rewriting steps are specified via CHR rules which replace a multi-set of constraints matching the left-hand side of a rule (also known as rule head) by the rule's right-hand side (also known as rule body).

CHR support a very fine-grained form of concurrency. CHR rules can be applied concurrently if the rewriting steps they imply do not interfere with each other. An interesting feature of CHR is that the left-hand side of a CHR rule can have a mix of simplified and propagated constraint patterns. This provides the opportunity for further concurrency. We can execute CHR rules concurrently as long as only their propagated parts overlap.

The fact that the abstract CHR semantics is highly concurrent has so far not been 
exploited in any major CHR implementation. Existing implementations are specified by highly deterministic semantics (Duck et al. 2004, De Koninck et al. 2008) which support efficient and systematic but inherently single-threaded execution schemes (Duck 2005, Schrijvers 2005). Our goal is to develop a systematic, yet concurrent, semantics which can be efficiently executed in parallel on a multi-core architecture. In the CHR context, there is practically no prior work which addresses this important issue.

Specifically, we make the following contributions:

- We develop a novel goal-based concurrent CHR semantics.

- We verify that our semantics respects the abstract CHR semantics by establishing precise correspondence results.

- We examine which existing sequential CHR optimizations carry over to the concurrent setting.

Section 4 contains the details. A concrete parallel implementation derived from our concurrent semantics is studied elsewhere (Sulzmann and Lam 2008). Section 5.2 provides a summary.

The upcoming section gives an overview of our work. Section 3 reviews the abstract CHR semantics. Section 5 discusses prior work on execution schemes for CHR and production rule systems which are a related rewriting mechanism. Section 6 concludes.

\section{Overview}

We first motivate concurrent execution of CHR rules via a few examples. Then, we review existing deterministic CHR execution schemes which are the basis for our concurrent goal-based CHR semantics.

\subsection{CHR and Concurrency}

Figures 1 and 2 contain several examples of CHR rules and derivations. We adopt the convention that lower-case symbols refer to variables and upper-case symbols refer to constraints. The notation rulename@ assigns distinct labels to CHR rules.

The first example simulates a simple communication channel. The Get $(x)$ constraint represents the action of writing a value from the communication channel into the variable $x$, while the $P u t(y)$ constraint represents the action of putting the value $y$ into the channel. The interaction between both constraints is specified via the CHR rule get which specifies the replacement of constraints matching Get $(x)$ and $\operatorname{Put}(y)$ by $x=y$. The point to note is that in contrast to Prolog, we use matching and not unification to trigger rules.

For example, the constraint store $\{G e t(m), P u t(1)\}$ matches the left-hand side of the get rule by instantiating $x$ by $m$ and $y$ by 1 . Hence, $\{\operatorname{Get}(m), P u t(1)\}$ rewrites to the answer $\{m=1\}$. We write $\{\operatorname{Get}(m), \operatorname{Put}(1)\} \longmapsto$ get $\{m=1\}$ to denote this derivation step. Similarly, we find that $\{\operatorname{Get}(n), P u t(8)\} \longmapsto$ get $\{n=8\}$. Rules can be applied concurrently as long as they do not interfere. In our case, the two 
Communication channel:

$$
\begin{gathered}
\text { get } @ \operatorname{Get}(x), \operatorname{Put}(y) \Longleftrightarrow x=y \\
\frac{\{\operatorname{Get}(m), \operatorname{Put}(1)\} \longmapsto \text { get }\{m=1\} \|\{\operatorname{Get}(n), \operatorname{Put}(8)\} \longmapsto_{\text {get }}\{n=8\}}{\{\operatorname{Get}(m), \operatorname{Put}(1), \operatorname{Get}(n), \operatorname{Put}(8)\} \longmapsto^{*}\{m=1, n=8\}}
\end{gathered}
$$

Greatest common divisor:

$\operatorname{gcd}_{1} @ \operatorname{Gcd}(0) \Longleftrightarrow$ True

$\operatorname{gcd} 2 @ \operatorname{Gcd}(n) \backslash G c d(m) \Longleftrightarrow m \geq n \& \& n>0 \mid G c d(m-n)$

$$
\{\operatorname{Gcd}(3), \operatorname{Gcd}(9)\} \longmapsto \operatorname{gcd} 2\{\operatorname{Gcd}(3), \operatorname{Gcd}(6)\}
$$

$\|$

$$
\{\operatorname{Gcd}(3), \operatorname{Gcd}(3)\} \longmapsto \operatorname{gcd} 2\{\operatorname{Gcd}(3), \operatorname{Gcd}(0)\}
$$

\begin{tabular}{rll}
\hline$\{\operatorname{Gcd}(3), \operatorname{Gcd}(3), \operatorname{Gcd}(9)\}$ & $\mapsto_{g c d 2, g c d 2}$ & $\{\operatorname{Gcd}(3), \operatorname{Gcd}(0), \operatorname{Gcd}(6)\}$ \\
& $\longmapsto^{*}$ & $\{\operatorname{Gcd}(3)\}$ \\
\hline
\end{tabular}

$$
\{\operatorname{Gcd}(3), \operatorname{Gcd}(3), \operatorname{Gcd}(9)\} \longmapsto^{*}\{\operatorname{Gcd}(3)\}
$$

Fig. 1. Communication channel and greatest common divisor

derivations above can be concurrently executed, indicated by the symbol $\|$, and we can straightforwardly combine both derivations which leads to the final answer $\{m=1, n=8\}$. We write $\longmapsto^{*}$ to denote exhaustive rule application.

The answer $\{m=8, n=1\}$ is also possible but the CHR rewrite semantics is committed-choice. We can guarantee a unique answer if the CHR rules are confluent which means that rewritings applicable on overlapping constraint sets are always joinable. In general, (non)confluence is of no concern to us here and is left to the programmer (if desired). We follow here the abstract CHR semantics (Frühwirth 1998) (formally defined in Section 3) which is inherently indeterministic. Rewrite rules can be applied in any order and thus CHR enjoy a high degree of concurrency.

The key to concurrency in CHR is monotonicity which guarantees that CHR executions remain valid if we include a larger context (i.e. store). The following result has been formally verified in (Abdennadher et al. 1999),

Theorem 1 (Monotonicity of $\mathrm{CHR}$ )

For any sets of CHR constraints $A, B$ and $S$, if $A \longmapsto^{*} B$ then $A \uplus S \longmapsto^{*} B \uplus S$

An immediate consequence of monotonicity is that concurrent CHR executions are sound in the sense that their effect can be reproduced using an appropriate sequential sequence of execution steps. Thus, we can derive the following rule:

$$
\text { (Concurrency) } \frac{S \uplus S_{1} \longmapsto^{*} S \uplus S_{2} \quad S \uplus S_{3} \longmapsto^{*} S \uplus S_{4}}{S \uplus S_{1} \uplus S_{3} \longmapsto^{*} S \uplus S_{2} \uplus S_{4}}
$$


Edmund S. L. Lam and Martin Sulzmann

$$
\begin{aligned}
& \text { merge1@Leq }(x, a) \backslash \operatorname{Leq}(x, b) \Longleftrightarrow a<b \mid \operatorname{Leq}(a, b) \\
& \text { merge } 2 @ \operatorname{Merge}(n, a), \operatorname{Merge}(n, b) \Longleftrightarrow a<b \mid \operatorname{Leq}(a, b), \operatorname{Merge}(n+1, a) \\
& \text { Shorthands: } L=L e q \text { and } M=\text { Merge } \\
& \begin{array}{ll} 
& M(1, a), M(1, c), M(1, e), M(1, g) \\
\longmapsto_{\text {merge } 2} & M(2, a), M(1, c), M(1, e), L(a, g) \\
\longmapsto_{\text {merge } 2} & M(2, a), M(2, c), L(a, g), L(c, e) \\
\longmapsto_{\text {merge } 2} & M(3, a), L(a, g), L(c, e), L(a, c) \\
\longmapsto_{\text {merge } 1} & M(3, a), L(a, c), L(c, g), L(c, e) \\
\longmapsto_{\text {merge } 1} & M(3, a), L(a, c), L(c, e), L(e, g)
\end{array}
\end{aligned}
$$

\begin{tabular}{|c|c|}
\hline & $M(3, a), L(a, c), L(c, e), L(e, g), M(3, b), L(b, d), L(d, f), L(f, h)$ \\
\hline$\longmapsto_{\text {merge } 2}$ & $M(4, a), L(a, c), L(a, b), L(c, e), L(e, g), L(b, d), L(d, f), L(f, h)$ \\
\hline$\longmapsto$ merge 1 & $M(4, a), L(a, b), L(b, c), L(c, e), L(e, g), L(b, d), L(d, f), L(f, h)$ \\
\hline$\longmapsto_{\text {merge } 1}$ & $M(4, a), L(a, b), L(b, c), L(c, d), L(c, e), L(e, g), L(d, f), L(f, h)$ \\
\hline$\longmapsto_{\text {merge } 1}$ & $M(4, a), L(a, b), L(b, c), L(c, d), L(d, e), L(e, g), L(d, f), L(f, h)$ \\
\hline$\longmapsto_{\text {merge } 1}$ & $M(4, a), L(a, b), L(b, c), L(c, d), L(d, e), L(e, f), L(e, g), L(f, h)$ \\
\hline$\longmapsto_{\text {merge } 1}$ & $M(4, a), L(a, b), L(b, c), L(c, d), L(d, e), L(e, f), L(f, g), L(f, h)$ \\
\hline$\longmapsto_{\text {merge } 1}$ & $M(4, a), L(a, b), L(b, c), L(c, d), L(d, e), L(e, f), L(f, g), L(g, h)$ \\
\hline
\end{tabular}

$$
\begin{aligned}
& M(1, b), M(1, d), M(1, f), M(1, h) \\
\longmapsto^{*} \quad & M(3, b), L(b, d), L(d, f), L(f, h)
\end{aligned}
$$

Fig. 2. Merge sort

In (Frühwirth 2005), the above is referred to as "Strong Parallelism of CHR". However, we prefer to use the term "concurrency" instead of "parallelism". In the CHR context, concurrency means to run a CHR program (i.e. a set of CHR rules) by using concurrent execution threads.

Let's consider the second CHR example from Figure1 which computes the greatest common divisor among a set of numbers by applying Euclid's algorithm. The left-hand side of rule $g c d 2$ is interesting because it uses a mix of simplified and propagated constraint patterns. We replace (simplify) $G c d(m)$ by $G c d(m-n)$ but keep (propagate) $G c d(n)$ if the guard $m \geq n \& \& n>0$ holds. For example, we find that $\{G c d(3), G c d(9)\} \longmapsto \operatorname{gcd} 2\{G c d(3), G c d(6)\}$ and $\{G c d(3), G c d(3)\} \longmapsto \operatorname{gcd} 2$ $\{G c d(3), G c d(0)\}$. The point to note is the above rule applications only overlap on the propagated part. Hence, we can execute both rewrite derivations simultaneously

$$
\{\operatorname{Gcd}(3), \operatorname{Gcd}(3) \operatorname{Gcd}(9)\} \longmapsto_{2 \times \operatorname{gcd} 2}\{\operatorname{Gcd}(3), \operatorname{Gcd}(\mathrm{o}), \operatorname{Gcd}(6)\}
$$


Our last example in Figure 2 is a CHR encoding of the well-known merge sort algorithm. To sort a sequence of (distinct) elements $e_{1}, \cdots, e_{m}$ where $m$ is a power of 2 , we apply the rules to the initial constraint store

$$
\operatorname{Merge}\left(1, e_{1}\right), \cdots, \operatorname{Merge}\left(1, e_{m}\right)
$$

Constraint $\operatorname{Merge}(n, e)$ refers to a sorted sequence of numbers at level $n$ whose smallest element is $e$. Constraint $\operatorname{Leq}(a, b)$ denotes that $a$ is less than $b$. Rule mergez initiates the merging of two sorted lists and creates a new sorted list at the next level. The actual merging is performed by rule merge1. Sorting of sublists belonging to different mergers can be performed simultaneously. See the example derivation in Figure 2 where we simultaneously sort the characters $a, c, e, g$ and $b, d, f, h$.

\subsection{Goal-Based CHR Execution}

Existing CHR implementation employ a more systematic CHR execution model where rules are triggered based on a set of available goals. The idea behind a goalbased CHR execution model is to separate the constraint store into two components: a set of goal constraints (constraints yet to be executed) and the actual constraint store (constraints that were executed). Previously, in the abstract semantics transitions $\longrightarrow_{\mathcal{A}}$ are among states Store whereas in the goal-based semantics we find now transitions $\longrightarrow_{\mathcal{G}}$ among states of the form $\langle$ Goals $|$ Store $\rangle$. Only goal constraints can trigger rules by searching for store constraint to build a complete match for a rule head, thus allowing for execution of the rule.

Below, we give a goal-based execution of the earlier communication buffer example.

\begin{tabular}{|c|c|c|}
\hline (D1 Activate) & $\longmapsto \mathcal{G}$ & $\begin{array}{l}\left\langle\left\{\operatorname{Get}\left(x_{1}\right), \operatorname{Get}\left(x_{2}\right), \operatorname{Put}(1), \operatorname{Put}(2)\right\} \mid\{\}\right\rangle \\
\left\langle\left\{\operatorname{Get}\left(x_{1}\right) \# 1, \operatorname{Get}\left(x_{2}\right), \operatorname{Put}(1), \operatorname{Put}(2)\right\} \mid\left\{\operatorname{Get}\left(x_{1}\right) \# 1\right\}\right\rangle\end{array}$ \\
\hline (D2 Drop) & $\longmapsto \mathcal{G}$ & $\left\langle\left\{\operatorname{Get}\left(x_{2}\right), \operatorname{Put}(1), \operatorname{Put}(2)\right\} \mid\left\{\operatorname{Get}\left(x_{1}\right) \# 1\right\}\right\rangle$ \\
\hline (D3 Activate) & $\longmapsto \mathcal{G}$ & $\left\langle\left\{\operatorname{Get}\left(x_{2}\right) \# 2, \operatorname{Put}(1), \operatorname{Put}(2)\right\} \mid\left\{\operatorname{Get}\left(x_{1}\right) \# 1, \operatorname{Get}\left(x_{2}\right) \# 2\right\}\right\rangle$ \\
\hline (D4 Drop) & $\longmapsto \mathcal{G}$ & $\left\langle\{\operatorname{Put}(1), \operatorname{Put}(2)\} \mid\left\{\operatorname{Get}\left(x_{1}\right) \# 1, \operatorname{Get}\left(x_{2}\right) \# 2\right\}\right\rangle$ \\
\hline (D5 Activate) & $\longmapsto \mathcal{G}$ & $\left\langle\{\operatorname{Put}(1) \# 3, \operatorname{Put}(2)\} \mid\left\{\operatorname{Get}\left(x_{1}\right) \# 1, \operatorname{Get}\left(x_{2}\right) \# 2, \operatorname{Put}(1) \# 3\right\}\right\rangle$ \\
\hline (D6 Fire get) & $\longmapsto_{\mathcal{G}}$ & $\left\langle\left\{\operatorname{Put}(2), x_{1}=1\right\} \mid\left\{\operatorname{Get}\left(x_{2}\right) \# 2\right\}\right\rangle$ \\
\hline (D7 Activate & $\succ_{\mathcal{G}}$ & $\left\langle\left\{\operatorname{Put}(2) \# 3, x_{1}=1\right\} \mid\left\{\operatorname{Get}\left(x_{2}\right) \# 2, \operatorname{Put}(2) \# 3\right\}\right\rangle$ \\
\hline (D8 Fire get) & $\longmapsto \mathcal{G}$ & $\left\langle\left\{x_{1}=1, x_{2}=2\right\} \mid\{\}\right\rangle$ \\
\hline (D9 Solve) & $\longmapsto \mathcal{G}$ & $\left\langle\left\{x_{2}=2\right\} \mid\left\{x_{1}=1\right\}\right\rangle$ \\
\hline (D10 Solve) & $\longmapsto \mathcal{G}$ & $\left\langle\{\} \mid\left\{x_{1}=1, x_{2}=2\right\}\right\rangle$ \\
\hline
\end{tabular}

We label the $x^{t h}$ derivation step by a label $D x$. Let's walk through each of the individual goal-based execution steps. Initially, all constraints are kept in the set of goals. At this point, all of the goals are inactive. Execution of goals proceeds in two stages: (1) Activation and (2a) rule execution, or (2b) dropping of goals. In the first stage, we activate a goal. In general, the order in which goals are activated is arbitrary. For concreteness, we assume a left-to-right activation order.

Hence, we first activate $\operatorname{Get}\left(x_{1}\right)$ in derivation step (D1). Active goals carry a 
unique identifier, a distinct integer number. Besides assigning numbers to active goals, we also put them into the store. For instance, after activating $\operatorname{Get}\left(x_{1}\right)$, we have $\operatorname{Get}\left(x_{1}\right) \# 1$ in both the goals and the store. 1

Active goals like $\operatorname{Get}\left(x_{1}\right) \# 1$ are executed by trying to build a complete match for a rule head with matching partner constraints in the store. Since there are no other constraints in the store, we cannot match $\operatorname{Get}\left(x_{1}\right) \# 1$ with the get rule. Therefore we drop Get $\left(x_{1}\right) \# 1$ in step (D2). Dropping of a goal means the goal is removed from the set of goals but of course the (now inactive) goal is still present in the store. Step (D3) and (D4) are similar but executed on goal $\operatorname{Get}\left(x_{2}\right)$. Then, we activate Get $\left(x_{2}\right)$ and find that $\operatorname{Get}\left(x_{2}\right) \# 2$ cannot build a complete match of the get rule, thus it is dropped too.

Next, we activate Put(1) (Step D5). Constraint Put(1)\#3 can match with either $\operatorname{Get}\left(x_{1}\right) \# 1$ or $\operatorname{Get}\left(x_{2}\right) \# 2$ to form a complete instance of rule head of get. We pick Get $\left(x_{1}\right) \# 1$ and fire the rule get, see step (D6). Step (D7) and (D8) perform similar execution steps on Put (2) and the remaining stored constraint Get $\left(x_{2}\right) \# 2$. Finally, we add the equations $x_{1}=1$ and $x_{2}=2$ into the store in steps (D9) and (D10). Exhaustive application of this goal-based execution strategy then leads to a state with no goals and a final store.

What we have described so far is essentially the execution scheme in which all major CHR implementations are based on. The semantics of these implementations assume a deterministic activation policy. For example, goals are kept in a stack (Duck et al. 2004) or priority queue (De Koninck et al. 2008). This of course implies a strictly sequential execution scheme.

To obtain a systematic, yet concurrent, CHR execution scheme we adapt the goalbased CHR semantics as follows. Several active goal constraints can simultaneously seek for partner constraints in the store to fire a rule instance. In the extreme case, all goal constraints could be activated at once. However, we generally assume that the number of active goals are bounded by $n$ where $n$ corresponds to the the number of actual threads available to the run-time system (for example, processor cores).

Figure 3 shows a sample concurrent goal-based CHR derivation. We assume two concurrent threads, referred to as $a$ and $b$, each thread executes the standard goalbased derivation steps. The novelty is that each goal-based derivation step $\stackrel{\delta}{\longmapsto} \mathcal{G}$ now records its effect on the store. The effect $\delta$ represents the sets of constraints in

\footnotetext{
${ }^{1}$ Numbered constraints also disambiguate multiple copies in the store but this is rather a sideeffect. The main purpose of numbering constraints is to indicate activation and retain the link between active goal constraints and their stored copy.
} 


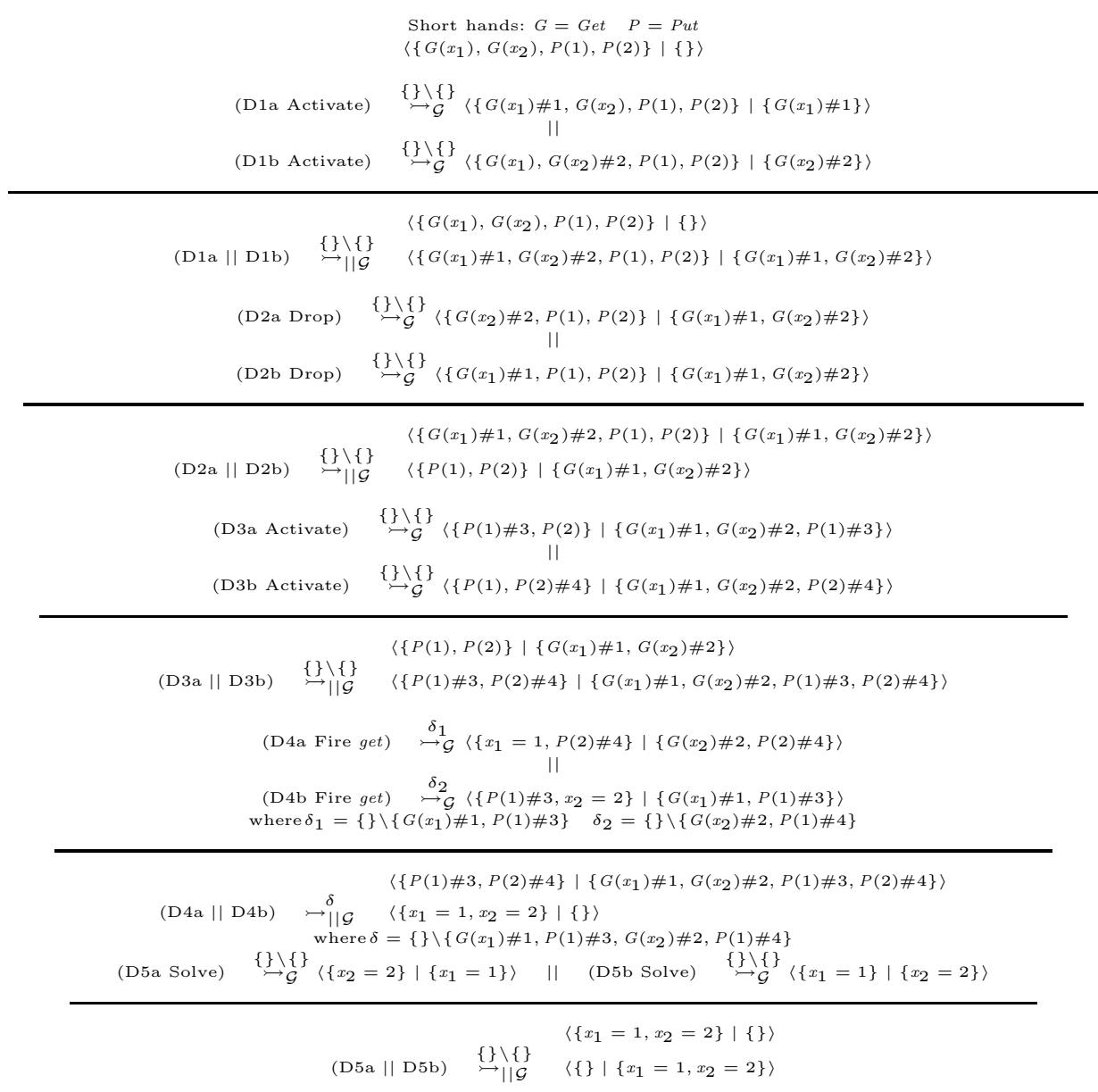

Fig. 3. Example of concurrent goal-based CHR derivation

the store which were propagated or simplified. Goal-based derivation steps can be executed concurrently if their effects are not in conflict.

$$
\begin{aligned}
& \left\langle G_{1} \mid H_{S 1} \cup H_{S 2} \cup S\right\rangle \stackrel{\delta_{1}}{\mapsto \| \mathcal{G}}\left\langle G_{1}^{\prime} \mid H_{S 2} \cup S\right\rangle \\
& \left\langle G_{2} \mid H_{S 1} \cup H_{S 2} \cup S\right\rangle \stackrel{\delta_{2}}{\boldsymbol{\delta}_{\| \mathcal{G}}}\left\langle G_{2}^{\prime} \mid H_{S 1} \cup S\right\rangle \\
& \delta_{1}=H_{P 1} \backslash H_{S 1} \quad \delta_{2}=H_{P 2} \backslash H_{S 2} \\
& \text { (Goal-Concurrency) } \quad H_{P 1} \subseteq S \quad H_{P 2} \subseteq S \quad \delta=H_{P 1} \cup H_{P 2} \backslash H_{S 1} \cup H_{S 2} \\
& \left\langle G_{1} \uplus G_{2} \uplus G \mid H_{S 1} \cup H_{S 2} \cup S\right\rangle \\
& \stackrel{\delta}{\longmapsto} \| \mathcal{G} \quad\left\langle G_{1}^{\prime} \uplus G_{2}^{\prime} \uplus G \mid S\right\rangle
\end{aligned}
$$

The (Goal-Concurrency) rule, abbreviated $(\| \mathcal{G})$, states that two goal-derivations are not in conflict if their simplification effects are disjoint and the propagated effects are present in the joint store. We will provide more explanations later. Let's continue with our example. 
Each thread activates one of the two Get goals (Steps D1a and D1b). Since both steps involve no rule application, side-effects are empty $(\{\} \backslash\{\})$. Both steps are executed concurrently denoted by the concurrent derivation step (D1a ||D2a) $\stackrel{\{\} \backslash\{\}}{\longmapsto}$. Concurrent goal-based execution threads operate on a shared store and their effects will be immediately made visible to other threads. This is important to guarantee exhaustive rule firings.

In the second step (D2a ||D2b), both active goals are dropped because there is no complete match for any rule head yet. Next, steps (D3a) and (D3b) activate the last two goal constraints, Put(1) and Put(2). Each active constraint can match with either of the two Get constraints in the store. We assume that active constraint Put(1)\#3 in step (D4a) matches with Get( $\left.x_{1}\right) \# 1$, while Put(2)\#4 in step (D4b) matches with $\operatorname{Get}\left(x_{2}\right) \# 2$, corresponding to the side-effects $\delta_{1}$ and $\delta_{2}$. This guarantees that steps (D4a) and (D4b) operates on different (non-conflicting) parts of the store. Thus, we can execute them concurrently which yields step (D4a||D4b). Their side-effects are combined as $\delta$. Finally, in step (D5a||D5b) we concurrently solve the two remaining equations by adding them into the store and we are done.

The correctness of our concurrent goal-based semantics is established by showing that all concurrent derivations can be replicated by sequential goal-based executions. We also prove that there is a correspondence between our goal-based CHR semantics with the abstract CHR semantics. This proof generalizes from (Duck 2005) which shows a correspondence between the refined CHR operational semantics and abstract semantics. There are a number of subtle points we came across when developing the concurrent variant of the goal-based semantics. We will postpone a discussion of these issues, as well as a complete formalization of the concurrent goal-based semantics until Section 4. Next, we formally introduce the details of the abstract CHR semantics.

\section{Constraint Handling Rules}

Figure 4 reviews the essentials of the abstract CHR semantics (Frühwirth 1998). The general form of CHR rules contains propagated heads $H_{P}$ and simplified heads $H_{S}$ as well as a guard $t_{g}$

$$
r @ H_{P} \backslash H_{S} \Longleftrightarrow t_{g} \mid B
$$

In CHR terminology, a rule with simplified heads only ( $H_{P}$ is empty) is referred to as a simplification rule, a rule with propagated heads only ( $H_{S}$ is empty) is referred to as a propagation rule. The general form is referred to as a simpagation rule.

CHR rules manipulate a global constraint store which is a multi-set of constraints. We execute CHRs by exhaustive rewriting of constraints in the store with respect to the given rule system (a finite set of CHR rules), via the derivations $\longmapsto$. To avoid ambiguities, we annotate derivations of the abstract semantics with $\mathcal{A}$.

Rule (Rewrite) describes application of a CHR rule $r$ at some instance $\phi$. We simply (remove from the store) the matching copies of $\phi\left(H_{S}\right)$ and propagate (keep in the

store) the matching copies of $\phi\left(H_{P}\right)$. But this only happens if the instantiated guard $\phi\left(t_{g}\right)$ is entailed by the equations present in the store $S$, written $\operatorname{Eqs}(S) \models \phi\left(t_{g}\right)$. 
Notations:

$\begin{array}{ll}\uplus & \text { Multi-set union } \\ \models & \text { Theoretic entailment } \\ \phi & \text { Substitution } \\ \bar{a} & \text { Set/List of } a \text { 's }\end{array}$

CHR Syntax:

$\begin{array}{ll}\text { Functions } & f::=+|>| \& \& \mid \cdots \\ \text { Constants } & v::=1 \mid \text { true } \mid \cdots \\ \text { Terms } & t::=x \mid f \bar{t} \\ \text { Predicates } & p::=\text { Get } \mid \text { Put } \mid \cdots \\ \text { Equations } & e::=t=t \\ \text { CHR constraints } & c::=p(\bar{t}) \\ \text { Constraints } & b::=e \mid c \\ \text { CHR Guards } & t_{g}::=t \\ \text { CHR Heads } & H::=\bar{c} \\ \text { CHR Body } & B::=\bar{b} \\ \text { CHR Rule } & R::=r @ H \backslash H \Longleftrightarrow t_{g} \mid B \\ \text { CHR Store } & S::=\bar{b} \\ \text { CHR Program } & \mathcal{P}::=\bar{R}\end{array}$

Abstract Semantics Rules: $\quad$ Store $\longmapsto \mathcal{A}$ Store

$\exists \phi \quad E q s(S) \models \phi \wedge t_{g} \quad \phi\left(H_{P} \uplus H_{S}\right)=H_{P}^{\prime} \uplus H_{S}^{\prime}$

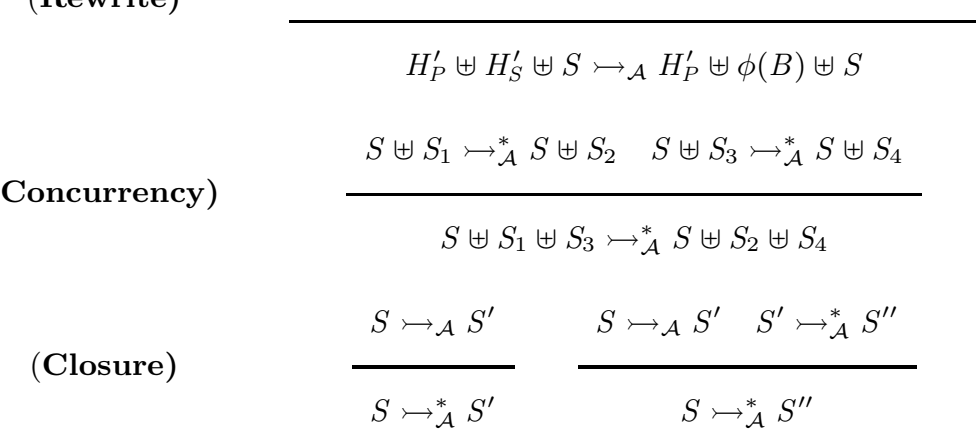

where $\operatorname{Eqs}(S)=\{e \mid e \in S, e$ is an equation $\}$

Fig. 4. Abstract CHR semantics

In case of a propagation rule we need to avoid infinite re-propagation. We refer to (Abdennadher 1997, Duck 2005) for details. Rule (Concurrency), introduced in (Frühwirth 2005), states that rules can be applied concurrently as long as they simplify on non-overlapping parts of the store.

Definition 3.1 (Non-overlapping Rule Application)

Two applications of the rule instances $r @ H_{P} \backslash H_{S} \Longleftrightarrow t_{g} \mid B$ and $r^{\prime} @ H_{P}^{\prime} \backslash H_{S}^{\prime} \Longleftrightarrow$ 


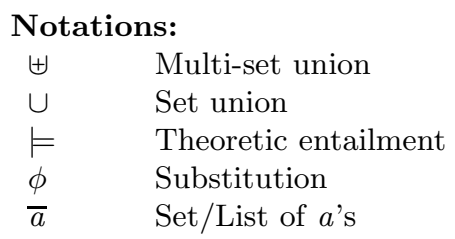

$\begin{array}{ll}\text { CHR Syntax: } & \\ \text { Functions } & f::=+|>| \& \& \mid \cdots \\ \text { Constants } & v::=1 \mid \text { true } \mid \cdots \\ \text { Terms } & t:=x \mid f \bar{t} \\ \text { Predicates } & p::=\text { Get } \mid \text { Put } \mid \cdots \\ \text { Equations } & e::=t=t \\ \text { CHR Constraints } & c:=p(\bar{t}) \\ \text { Constraints } & b:=e \mid c \\ \text { CHR Guards } & t_{g}::=t \\ \text { CHR Heads } & H::=\bar{c} \\ \text { CHR Body } & B::=\bar{b} \\ \text { CHR Rule } & R::=r @ H \backslash H \Longleftrightarrow t_{g} \mid B \\ \text { CHR Program } & \mathcal{P}::=\bar{R} \\ \text { Num Constraint } & n c::=c \# i \\ \text { Goal Constraint } & g::=c|e| n c \\ \text { Stored Constraint } & s c::=n c \mid e \\ \text { CHR Num Store } & S n::=\overline{s c} \\ \text { CHR Goals } & G::=\bar{g} \\ \text { CHR State } & \sigma::=\langle G, S n\rangle \\ \text { Side Effects } & \delta::=S n \backslash S n\end{array}$

Fig. 5. CHR Goal-based Syntax

$t_{g}^{\prime} \mid B^{\prime}$ in store $S$ are said to be non-overlapping if and only if they simplify unique parts of $S$ (i.e. $H_{S}, H_{S}^{\prime} \subseteq S$ and $H_{S} \cap H_{S}^{\prime}=\emptyset$ ).

The two last (Closure) rules simply specify the transitive application of CHR rules.

\section{Concurrent Goal-Based CHR Operational Semantics}

We present the formal details of the concurrent goal-based CHR semantics. Figure 5 describes the necessary syntactic extensions. Because constraints in the store now have unique identifiers, we treat the store as a set (as opposed to a multiset) and use set union $\cup$. Goals are still treated as multi-sets because they can contain multiple copies of (un-numbered) CHR constraints. The actual semantics is given in two parts. Figure 6 describes the single-step execution part whereas Figure 7 introduces the concurrent execution part. The first part is a generalization of an earlier goal-based description (Duck 2005) whereas the second (concurrent) part is novel.

We first discuss the single-step derivation steps in Figure 6, A derivation step $\sigma \stackrel{\delta}{\longmapsto} \mathcal{G} \sigma^{\prime}$ maps the CHR state $\sigma$ to $\sigma^{\prime}$ with some side-effect $\delta$. $\delta$ represents the con- 
Concurrent Goal-Based Execution of Constraint Handling Rules

$$
\langle\text { Goal }| \text { Store }\rangle \stackrel{\delta}{\stackrel{\delta}{\rightarrow}}\langle\text { Goal }| \text { Store }\rangle
$$

(Solve)

$$
W=W a k e U p(e, S n)
$$

$$
\langle\{e\} \uplus G \mid S n\rangle \stackrel{W \backslash\{\}}{\longmapsto \mathcal{G}}\langle W \uplus G \mid\{e\} \cup S n\rangle
$$

$i$ is a fresh identifier

(Activate)

(Simplify)

$$
\begin{gathered}
\langle\{c\} \uplus G \mid S n\rangle \stackrel{\{\} \backslash\{\}}{\mapsto \mathcal{G}}\langle\{c \# i\} \uplus G \mid\{c \# i\} \cup S n\rangle \\
\left(r @ H_{P}^{\prime} \backslash H_{S}^{\prime} \Longleftrightarrow t_{g} \mid B^{\prime}\right) \in \mathcal{P} \text { such that } \\
\exists \phi \quad E q s(S n) \models \phi \wedge t_{g} \quad \phi\left(H_{P}^{\prime}\right)=\operatorname{DropIds}\left(H_{P}\right) \\
\phi\left(H_{S}^{\prime}\right)=\phi\left(\{c\} \uplus \operatorname{DropIds}\left(H_{S}\right)\right) \\
\delta=H_{P} \backslash\{c \# j\} \cup H_{S}
\end{gathered}
$$

$$
\begin{gathered}
\left\langle\{c \# j\} \uplus G \mid\{c \# j\} \cup H_{P} \cup H_{S} \cup S n\right\rangle \\
\stackrel{\delta}{\longmapsto} \mathcal{G} \quad\left\langle\phi\left(B^{\prime}\right) \uplus G \mid H_{S} \cup S n\right\rangle \\
\quad\left(r @ H_{P}^{\prime} \backslash H_{S}^{\prime} \Longleftrightarrow t_{g} \mid B^{\prime}\right) \in \mathcal{P} \text { such that } \\
\exists \phi \quad E q s(S n) \models \phi \wedge t_{g} \quad \phi\left(H_{S}^{\prime}\right)=\operatorname{DropIds}\left(H_{S}\right) \\
\phi\left(H_{P}^{\prime}\right)=\phi\left(\{c\} \uplus \operatorname{DropIds}\left(H_{P}\right)\right) \\
\delta=\{c \# j\} \cup H_{P} \backslash H_{S} \\
\stackrel{\delta}{\zeta \mathcal{G}} \quad\left\langle\phi\left(B^{\prime}\right) \uplus\{c \# j\} \uplus G \mid\{c \# j\} \cup H_{P} \cup S n\right\rangle
\end{gathered}
$$

(Propagate)

(Simplify) and (Propagate) does not apply on $c \# j$ in $S n$

$$
\begin{aligned}
\text { (Drop) } & \\
& \langle\{c \# j\} \uplus G \mid S n\rangle \stackrel{\{\} \backslash\{\}}{\longmapsto \mathcal{G}}\langle G \mid S n\rangle \\
\text { where } \operatorname{Eqs}(S) & \{e \mid e \in S, e \text { is an equation }\} \\
\operatorname{DropIds}(S n)= & \{c \mid c \# i \in S n\} \uplus\{e \mid e \in S n, e \text { is an equation }\} \\
\operatorname{WakeUp}(e, S n)= & \{c \# i \mid c \# i \in S n \wedge \phi \text { m.g.u. of Eqs }(S n) \wedge \\
& \theta \text { m.g.u. of Eqs }(S n \cup\{e\}) \wedge \phi(c) \neq \theta(c)\}
\end{aligned}
$$

Fig. 6. Goal-Based CHR Semantics (Single-Step Execution)

straints that where propagated or simplified during rule application. Hence derivation steps that do not involve rule application ((Activate) and (Drop)) contain no side-effects (i.e. \{\}$\backslash\{\})$. We will omit side-effects $\delta$ as and when it is not relevant to our discussions. We ignore the (Solve) step for the moment. In (Activate), we activate a goal CHR constraint by assigning it a fresh unique identifier and adding it to the store. Rewrite rules are executed in steps (Simplify) and (Propagate). We 
distinguish whether the rewrite rule is executed on a simplified or propagated active (goal) constraint $c \# i$. For both cases, we seek for the missing partner constraints in the store for some matching substitution $\phi$. The auxiliary function DropIds ignores the unique identifiers of numbered constraints. They do not matter when finding a rule head match. The guard $t_{g}$ must be entailed by the primitive (here equational) store constraints under the substitution $\phi$.

In case of a simplified goal, step (Simplify), we apply the rule instance of $r$ by deleting all simplified matching constraints $H_{S}$ and adding the rule body instance $\phi(B)$ into the goals. Since $c \# i$ is simplified, we drop $c \# i$ from the goals as it does not exist in the store any more. In case of a propagated goal, step (Propagate), $c \# i$ remains in the goal set as well in the store and thus can possibly fire further rules

instances. For both (Simplify) and (Propagate) derivation step, say $\sigma \stackrel{H_{P} \backslash H_{S}}{\overbrace{\mathcal{G}}} \sigma^{\prime}$, we record as side-effect the numbered constraints in the store that were propagated $\left(H_{P}\right)$ or simplified $\left(H_{S}\right)$ during the derivation step. We will elaborate on the purpose of side-effects when we introduce the concurrent part of the semantics.

In step (Drop), we remove an active constraint from the set of goals, if the constraint failed to trigger any CHR rule.

Rule (Solve) moves an equation goal $e$ into the store and wakes up (reactivates) any numbered constraint in the store which can possibly trigger further CHR rules due to the presence of $e$. Here is a simple example to show why reactivation is necessary.

$$
\begin{aligned}
& r 1 @ A(x), B(x) \Longleftrightarrow C(x) \\
& \begin{array}{lll}
\text { (Solve) } & \{A(2) \# 1\} \backslash\{\} & \langle\{A(2) \# 1\} \mid\{A(2) \# 1, B(2) \# 2, a=2\}\rangle \\
\text { (Simp } r 1) & \{\} \backslash\{A(2) \# 1, B(2) \# 2\} & \langle\{C(2)\} \mid\{a=2\}\rangle
\end{array} \\
& \text {... }
\end{aligned}
$$

For clarity, we normalize all constraints in the store once an equation is added. Prior to addition of $a=2, A(a) \# 1, B(2) \# 2$ cannot fire rule $r$. After adding $a=2$ however, we can normalize $A(a) \# 1$ to $A(2) \# 2$, which can now fire $r 1$ with $B(2) \# 2$. To guarantee exhaustive rule firings, we reactivate $A(2) \# 2$ by adding it back to the set of goals. WakeUp $(e, S n)$ represents a conservative approximation of the to be reactivated constraints (Duck 2005). Note that we treat reactivated constraints as propagated constraints in the side-effects.

Figure 7 presents the concurrent part of the goal-based operational semantics. In the (Lift) step, we turn a sequential goal-based derivation into a concurrent derivation. Note that side-effects are retained. Step (Goal Concurrency) joins together two concurrent derivations operating on a shared store, if their rewriting side-effects $\delta_{1}$ and $\delta_{2}$ are non-overlapping as defined below.

Definition 4.1 (Non-overlapping Rewriting Side-Effects)

Two rewriting side-effects $\delta_{1}=H_{P 1} \backslash H_{S 1}$ and $\delta_{2}=H_{P 2} \backslash H_{S 2}$ are said to be nonoverlapping, if and only if $H_{S 1} \cap\left(H_{P 2} \cup H_{S 2}\right)=\{\}$ and $H_{S 2} \cap\left(H_{P 1} \cup H_{S 1}\right)=\{\}$

Concurrent derivations with non-overlapping side-effects essentially simplify distinct constraints in the store, as well as propagate constraints which are not simpli- 
Concurrent Goal-Based Execution of Constraint Handling Rules

$$
\begin{aligned}
& \langle\text { Goal }| \text { Store }\rangle \stackrel{\delta}{\longmapsto} \| \mathcal{G}\langle\text { Goal }| \text { Store }\rangle \\
& \langle G \mid S n\rangle \stackrel{\delta}{\mapsto \mathcal{G}}\left\langle G^{\prime} \mid S n^{\prime}\right\rangle \\
& \text { (Lift) } \\
& \langle G \mid S n\rangle \stackrel{\delta}{\mapsto \| \mathcal{G}}\left\langle G^{\prime} \mid S n^{\prime}\right\rangle \\
& \left\langle G_{1} \mid H_{S 1} \cup H_{S 2} \cup S\right\rangle \stackrel{\delta_{1}}{\longmapsto} \| \mathcal{G}\left\langle G_{1}^{\prime} \mid H_{S 2} \cup S\right\rangle \\
& \left\langle G_{2} \mid H_{S 1} \cup H_{S 2} \cup S\right\rangle \stackrel{\delta_{2}}{\longmapsto}\left\langle G_{\mathcal{G}}\left\langle G_{2}^{\prime} \mid H_{S 1} \cup S\right\rangle\right. \\
& \delta_{1}=H_{P 1} \backslash H_{S 1} \quad \delta_{2}=H_{P 2} \backslash H_{S 2} \\
& \text { (Goal Concurrency) } \quad H_{P 1} \subseteq S \quad H_{P 2} \subseteq S \quad \delta=H_{P 1} \cup H_{P 2} \backslash H_{S 1} \cup H_{S 2} \\
& \left\langle G_{1} \uplus G_{2} \uplus G \mid H_{S 1} \cup H_{S 2} \cup S\right\rangle \\
& \stackrel{\delta}{\longmapsto} \| \mathcal{G} \quad\left\langle G_{1}^{\prime} \uplus G_{2}^{\prime} \uplus G \mid S\right\rangle \\
& \sigma \stackrel{\delta}{\mapsto \| \mathcal{G}} \sigma^{\prime} \quad \sigma \stackrel{\delta}{\mapsto \| \mathcal{G}} \sigma^{\prime} \quad \sigma^{\prime} \mapsto{ }_{\| \mathcal{G}}^{*} \sigma^{\prime \prime} \\
& \text { (Closure) } \\
& \overline{\sigma \longmapsto{ }_{\| \mathcal{G}}^{*} \sigma^{\prime}} \quad \sigma \longmapsto{ }_{\| \mathcal{G}}^{*} \sigma^{\prime \prime}
\end{aligned}
$$

Fig. 7. Goal-Based CHR Semantics (Concurrent Part)

fied by one another. The (Goal Concurrency) step expresses non-overlapping sideeffects by structurally enforcing that simplified constraints $H_{S 1}$ and $H_{S 2}$ match distinct parts of the store, while propagated constraints $H_{P 1}$ and $H_{P 2}$ are found in the shared part of the store $S$ not modified by both concurrent derivations. In the resulting concurrent derivation, the side-effects $\delta_{1}$ and $\delta_{2}$ are composed by the union of the propagate and simplify components respectively, forming $\delta$.

An immediate consequence is that we can execute $k$ derivations concurrently by stacking them together as long as all side-effects are mutually non-overlapping. The following lemma summarizes this observation.

Lemma 1 (k-Concurrency) 
For any finite $k$ of mutually non-overlapping concurrent derivations,

$$
\begin{gathered}
\left\langle G_{1} \mid H_{S 1} \cup \cdots \cup H_{S i} \cup \cdots \cup H_{S k} \cup S\right\rangle \stackrel{H_{P 1} \backslash H_{S 1}}{\longmapsto \| \mathcal{G}}\left\langle G_{1}^{\prime} \mid\{\} \cup \cdots \cup H_{S i} \cup \cdots \cup H_{S k} \cup S\right\rangle \\
\ldots \\
\left\langle G_{i} \mid H_{S 1} \cup \cdots \cup H_{S i} \cup \cdots \cup H_{S k} \cup S\right\rangle \stackrel{H_{P i} \backslash H_{S i}}{\longmapsto}\left\langle G_{i}^{\prime} \mid H_{S 1} \cup \cdots \cup\{\} \cup \cdots \cup H_{S k} \cup S\right\rangle \\
\cdots \\
\left\langle G_{k} \mid H_{S 1} \cup \cdots \cup H_{S i} \cup \cdots \cup H_{S k} \cup S\right\rangle \stackrel{H_{P k} \backslash H_{S k}}{\longmapsto}\left\langle G_{k}^{\prime} \mid H_{S 1} \cup \cdots \cup H_{S i} \cup \cdots \cup\{\} \cup S\right\rangle \\
H_{P 1} \subseteq S \cdots H_{P i} \subseteq S \cdot H_{P k} \subseteq S \\
\delta=H_{P 1} \cup \cdots \cup H_{P i} \cup \cdots \cup H_{P k} \backslash H_{S 1} \cup \cdots \cup H_{S i} \cup \cdots \cup H_{S k} \\
\quad\left\langle G_{1} \uplus \cdots \uplus G_{i} \uplus \cdots \uplus G_{k} \uplus G \mid H_{S 1} \cup \cdots \cup H_{S i} \cup \cdots \cup H_{S k} \cup S\right\rangle \\
\qquad \| \mathcal{G} \quad\left\langle G_{1}^{\prime} \uplus \cdots \uplus G_{i}^{\prime} \uplus \cdots \uplus G_{k}^{\prime} \uplus G \mid S\right\rangle
\end{gathered}
$$

we can decompose this into $k-1$ applications of the (pair-wise) (Goal Concurrency) derivation step.

The (Closure) step defines transitive application of the concurrent goal-based derivation. Because side-effect labels are only necessary for the (Goal Concurrency) step, we drop the side-effects in transitive derivations.

Any concurrent goal-based derivation can be reproduced in the abstract CHR semantics. This correspondence result is important to make use of the concurrent goal-based semantics as a more systematic execution scheme for CHR. We will formally verify this as well as other results in the up-coming Section 4.2. First, we give an in-depth discussion of the more subtle aspects of the concurrent goal-based semantics.

\subsection{Discussion}

Most of the issues we encounter are related to the problem of exhaustive rule firings. For brevity, we omit side-effects in derivation steps in the following examples as they do not matter.

Goal Storage, Shared Store and Single-Step Execution: Each of these issues affect (exhaustive) rule firings. We first consider goal storage. Suppose we would only store goals after execution (rule head matching). That is, we do not add the goals into the store during (Activate) step, but only during the (Drop) step.

$$
i \text { is a fresh identifier }
$$

\section{(Activate')}

$$
\langle\{c\} \uplus G \mid S n\rangle \mapsto_{\mathcal{G}}\langle\{c \# i\} \uplus G \mid S n\rangle
$$

(Simplify) and (Propagate) does not apply on $c \# i$ in $S n$

(Drop')

$$
\langle\{c \# i\} \uplus G \mid S n\rangle \mapsto \mathcal{G}\langle G \mid\{c \# i\} \cup S n\rangle
$$

Then, for the CHR program

$$
r 1 @ A(x), B(y) \Longleftrightarrow C(x, y)
$$


we obtain the following derivation

$$
\begin{gathered}
\langle\{A(1), B(2)\} \mid\{\}\rangle \\
(\text { Activate') }\langle\{A(1)\} \mid\{\}\rangle \longmapsto \| \mathcal{G}\langle\{A(1) \# 1\} \mid\{\}\rangle \\
\frac{\|}{(\text { Activate' }) \quad\langle\{B(2)\} \mid\{\}\rangle \longmapsto \| \mathcal{G}\langle\{B(2) \# 2\} \mid\{\}\rangle} \\
\langle\{A(1), B(2)\} \mid\{\}\rangle \longmapsto \| \mathcal{G}\langle\{A(1) \# 1, B(2) \# 2\} \mid\{\}\rangle \\
(\text { Drop') }\langle\{A(1) \# 1\} \mid\{\}\rangle \longmapsto \| \mathcal{G}\langle\{\} \mid\{A(1) \# 1\}\rangle \\
(\text { Drop') }\langle\{B(2) \# 2\} \mid\{\}\rangle \longmapsto \| \mathcal{G}\langle\{\} \mid\{B(2) \# 2\}\rangle \\
\hline\langle\{A(1) \# 1, B(2) \# 2\} \mid\{\}\rangle \longmapsto \| \mathcal{G}\langle\{\} \mid\{A(1) \# 1, B(2) \# 2\}\rangle
\end{gathered}
$$

Initially both goals $A(1)$ and $B(2)$ are concurrently activated. Since (Activate') does not store goals immediately, both active goals are not visible to each other in the store. Hence, we wrongfully apply the (Drop') step for both goals. However, there is clearly a complete rule head match $A(1) \# 1, B(2) \# 2$.

Next, we investigate the shared store issue. Suppose we allow for concurrent executions on (non-shared) split stores. Then, the following derivation is possible.

$$
\begin{aligned}
& r 1 @ A, B \Longleftrightarrow C \quad r 2 @ D, E \Longleftrightarrow F \\
& \text { (Drop) }\langle\{A \# 3\} \mid\{A \# 3, E \# 2\}\rangle \longmapsto \| \mathcal{G}\langle\{\} \mid\{A \# 3, E \# 2\}\rangle \\
& \text { (Drop) }\langle\{D \# 4\} \mid\{B \# 1, D \# 4\}\rangle \longmapsto \| \mathcal{G}\langle\{\} \mid\{B \# 1, D \# 4\}\rangle \\
& \langle\{A \# 3, D \# 4\} \mid\{A \# 3, B \# 1, D \# 4, E \# 2\}\rangle \longmapsto \| \mathcal{G}\langle\{\} \mid\{A \# 3, B \# 1, D \# 4, E \# 2\}\rangle
\end{aligned}
$$

The resulting store is a final store, there are no more goals left. However, if we consider the entire store $\{A \# 3, E \# 2, B \# 1, D \# 4\}$, it is clearly that goal $A \# 3$ can execute rule $r_{1}$ and goal $D \# 4$ can execute rule $r_{2}$. We conclude that splitting of the store leads to "stuck" states. We fail to exhaustively fire CHR rules.

For similar reasons, we demand that when joining concurrent executions, each individual execution can only make a single-step. Otherwise, we encounter again a stuck state.

$$
\begin{aligned}
& r 1 @ A, B \Longleftrightarrow C \\
& (P 1)\langle\{A\} \mid\{\}\rangle \hookrightarrow_{\| \mathcal{G}}\langle\{A \# 2\} \mid\{A \# 2\}\rangle \hookrightarrow_{\| \mathcal{G}}\langle\{\} \mid\{A \# 2\}\rangle \\
& (P 2)\langle\{B\} \mid\{\}\rangle \longmapsto \|_{\mathcal{G}}\langle\{B \# 3\} \mid\{B \# 3\}\rangle \longmapsto_{\| \mathcal{G}}\langle\{\} \mid\{B \# 3\}\rangle \\
& \langle\{A, B\} \mid\{\}\rangle \longmapsto_{\| \mathcal{G}}^{*}\langle\{\} \mid\{A \# 2, B \# 3\}\rangle
\end{aligned}
$$

The sequence of derivation steps (P1) first activates $A$ which is then dropped. Similarly, (P2) activates $B$ which is then dropped as well which then leads to the stuck state $\langle\{\} \mid\{A \# 2, B \# 3\}\rangle$. We clearly missed to fire rule $r 1$. This shows that single-step concurrent execution are essential to guarantee that newly added 
constraints are visible to all concurrent active goals, hence we have exhaustive rule firings in the goal-based semantics.

The underlying reason for non-exhaustive firing of rules is that the goal-based semantics is not monotonic in its store argument. However, execution is monotonic in the goal argument which leads us to the next issue.

Lazy Matching and Asynchronous Goal Execution: When executing goals, we lazily compute only matches that contain the specific goal and immediately apply such matches without concerning any further matches. For instance consider the following CHR program and goal-based derivation:

$$
\begin{gathered}
r 0 @ A(x), B(y) \Longleftrightarrow D(x, y) \\
\langle\{A(1) \# 4\} \uplus\{A(2), A(3)\} \mid\{B(2) \# 1, B(3) \# 2, B(4) \# 3, A(1) \# 4\}\rangle \\
\neg \mathcal{G} \quad\langle\{D(1,2)\} \uplus\{A(2), A(3)\} \mid\{B(3) \# 2, B(4) \# 3\}\rangle
\end{gathered}
$$

We have applied the rule instance $A(1) \# 4, B(2) \# 1$ independently of the existence of the other goals (i.e. $\{A(2), A(3)\})$. In the literature, such a matching scheme is known as a lazy matching scheme, and often implemented by variants of the LEAPS algorithm (D.P. Miranker and Gadbois 1990).

Lazy matching in the goal-based semantics is possible only because the goal-based semantics is monotonic with respect to the set of goals. The following illustrates this monotonicity property of goals:

$$
\begin{aligned}
& \langle\{A(1) \# 4\} \mid\{B(2) \# 1, B(3) \# 2, B(4) \# 3, A(1) \# 4\}\rangle \\
\longmapsto \mathcal{G} & \langle\{D(1,2)\} \mid\{B(3) \# 2, B(4) \# 3\}\rangle
\end{aligned}
$$

$\langle\{A(1) \# 4\} \uplus\{A(2), A(3)\} \mid\{B(2) \# 1, B(3) \# 2, B(4) \# 3, A(1) \# 4\}\rangle$
$\longmapsto \mathcal{G} \quad\langle\{D(1,2)\} \uplus\{A(2), A(3)\} \mid\{B(3) \# 2, B(4) \# 3\}\rangle$
$\frac{\langle G \mid S n\rangle r_{\mathcal{G}}\left\langle G^{\prime} \mid S n^{\prime}\right\rangle}{\left\langle G \uplus G^{\prime \prime} \mid S n\right\rangle r_{\mathcal{G}}\left\langle G^{\prime} \uplus G^{\prime \prime} \mid S n^{\prime}\right\rangle}$

The above property essentially states that we can execute goals $G$ without prior knowledge of goals $G^{\prime \prime}$. Because of monotonicity, we are guaranteed that future executions of $G^{\prime \prime}$ will not invalidate them. 
Monotonicity of the goals also allows us to execute goals asynchronously. For instance, consider the following:

$$
\begin{aligned}
& r 1 @ A(x), B(y) \Longleftrightarrow C(x, y) \\
& \langle\{A(1) \# 1\} \mid\{A(1) \# 1, B(2) \# 2\} \cup\{A(3) \# 3, B(4) \# 4\}\rangle \\
\delta_{1} & \langle\{C(1,2)\} \mid\{\} \cup\{A(3) \# 3, B(4) \# 4\}\rangle \\
& \langle\{A(3) \# 3\} \mid\{A(1) \# 1, B(2) \# 2\} \cup\{A(3) \# 3, B(4) \# 4\}\rangle \\
\delta_{2} & \langle\{C(3,4)\} \mid\{A(1) \# 1, B(2) \# 2\} \cup\{\}\rangle \\
\delta_{1}= & \{\} \backslash\{A(1) \# 1, B(2) \# 2\} \quad \delta_{2}=\{\} \backslash\{A(3) \# 3, B(4) \# 4\} \\
& \delta=\{\} \backslash\{A(1) \# 1, B(2) \# 2, A(3) \# 3, B(4) \# 4\}
\end{aligned}
$$

$$
\begin{aligned}
& \langle\{A(1) \# 1\} \uplus\{A(3) \# 3\} \mid\{A(1) \# 1, B(2) \# 2\} \cup\{A(3) \# 3, B(4) \# 4\}\rangle \\
& \stackrel{\delta}{\longmapsto} \| \mathcal{G} \quad\langle\{C(1,2)\} \uplus\{C(3,4)\} \mid\{\} \cup\{\}\rangle
\end{aligned}
$$

The above describes the concurrent execution of goals $A(1) \# 1$ and $A(3) \# 3$. Notice that in the derivations of the premise, we can ignore all goals which are not relevant to the derivation. For instance, execution of $A(1) \# 1$ does not need goal $A(3) \# 3$ to be visible, hence the goals effectively executes asynchronously. Goals do however, implicitly "synchronize" via the shared store. Namely, concurrent derivations must be chosen such that rewrite side-effects involve distinct parts of the store.

\subsection{Correspondence Results}

We formally verify that the concurrent goal-based semantics is in exact correspondence to the abstract CHR semantics when it comes to termination and exhaustive rule firings. Detailed proofs are given in the appendix. In the main text, we provide key lemmas and proof sketches. We first introduce some elementary definitions before stating the formal results.

Definitions: The first two definitions concern the abstract CHR semantics. A store is final if no further rules are applicable.

\section{Definition 4.2 (Final Store)}

A store $S$ is known as a final store, denoted $\operatorname{Final}_{\mathcal{A}}(S)$ if and only if no more CHR rules applies on it (i.e. $\neg \exists S^{\prime}$ such that $S \longmapsto_{\mathcal{A}} S^{\prime}$ ).

A CHR program terminates if all derivations lead to a final store in a finite number of states.

\section{Definition 4.3 (Terminating CHR Programs)}

A CHR program $\mathcal{P}$ is said to be terminating, if and only if for any CHR store $S$, all derivations starting from $S$ are finite.

Next, we introduce some definitions in terms of the goal-based semantics. In an initial state, all constraints are goals and the store is empty. Final states are states 
which no longer have any goals. We will prove the exhaustiveness of the goalbased semantics by proving a correspondence between final stores in the abstract semantics and final states of the goal-based semantics

\section{Definition 4.4 (Initial and Final CHR States)}

An initial CHR state is a CHR state of the form $\langle G \mid\{\}\rangle$ where $G$ contains no numbered constraints $(c \# n)$, while a final CHR state is of the form $\langle\{\} \mid S n\rangle$

A state is reachable if there exists a (sequential) goal-based sequence of derivations to this state. We write $\longmapsto_{\mathcal{G}}^{*}$ to denote the transitive closure of $\succ_{\mathcal{G}}$.

Definition 4.5 (Sequentially Reachable CHR states)

For any CHR program $\mathcal{P}$, a CHR state $\left\langle G^{\prime} \mid S n^{\prime}\right\rangle$ is said to be sequentially reachable by $\mathcal{P}$ if and only if there exists some initial CHR state $\langle G \mid\{\}\rangle$ such that $\langle G|$ \{\}$\rangle \longmapsto_{\mathcal{G}}^{*}\left\langle G^{\prime} \mid S n^{\prime}\right\rangle$.

\subsubsection{Correspondence of Derivations}

We build a correspondence between the abstract semantics and the concurrent goalbased semantics. We begin with Theorem 2, which states the correspondence of the (sequential) goal-based semantics.

Theorem 2 (Correspondence of Sequential Derivations)

For any reachable CHR state $\langle G \mid S n\rangle$, CHR state $\left\langle G^{\prime} \mid S n^{\prime}\right\rangle$ and CHR program $\mathcal{P}$,

$$
\begin{array}{ll}
\text { if } \quad & \langle G \mid S n\rangle \hookrightarrow_{\mathcal{G}}^{*}\left\langle G^{\prime} \mid S n^{\prime}\right\rangle \\
\text { then } & (\operatorname{NoIds}(G) \uplus \operatorname{DropIds}(S n))=\left(\operatorname{NoIds}\left(G^{\prime}\right) \uplus \operatorname{DropIds}\left(S n^{\prime}\right)\right) \quad \vee \\
& (\operatorname{NoIds}(G) \uplus \operatorname{DropIds}(S n)) \longmapsto_{\mathcal{A}}^{*}\left(\operatorname{NoIds}\left(G^{\prime}\right) \uplus \operatorname{DropIds}\left(S n^{\prime}\right)\right)
\end{array}
$$

where NoIds $=\{c \mid c \in G, c$ is a CHR constraint $\} \uplus\{e \mid e \in G, e$ is an equation $\}$

The above result guarantees that any sequence of sequential goal-based derivations starting from a reachable CHR state either yields equivalent CHR abstract stores (due to goal-based behavior not captured by the abstract semantics, namely (Solve) (Activate), (Drop)) or corresponds to a derivation in the abstract semantics (due to rule application). A goal-based semantics state $\langle G \mid S n\rangle$ is related to an abstract semantics store by removing all numbered constraints in $G$ and unioning it with constraints in $S n$ without their identifiers. The theorem and its proof is a generalization of an earlier result given in (Duck 2005).

We formalize the observation that the goal context can be extended without interfering with previous goal executions.

Lemma 2 (Monotonicity of Goals in Goal-based Semantics)

For any goals $G, G^{\prime}$ and $G^{\prime \prime}$ and CHR store $S n$ and $S n^{\prime}$, If $\langle G \mid S n\rangle \longmapsto_{\mathcal{G}}^{*}\left\langle G^{\prime} \mid S n^{\prime}\right\rangle$ then $\left\langle G \uplus G^{\prime \prime} \mid S n\right\rangle \longmapsto_{\mathcal{G}}^{*}\left\langle G^{\prime} \uplus G^{\prime \prime} \mid S n^{\prime}\right\rangle$.

Next, we state that given any goal-based derivation with side-effects $\delta$, we can safely ignore any constraints (represented by $S_{2}$ ) in the store which is not part of $\delta$. 
Lemma 3 (Isolation of Goal-based Derivations)

If $\left\langle G \mid H_{P} \cup H_{S} \cup S_{1} \cup S_{2}\right\rangle \stackrel{H_{P} \backslash H_{S}}{\longmapsto \mathcal{G}}\left\langle G^{\prime} \mid H_{P} \cup S_{1}^{\prime} \cup S_{2}\right\rangle$

then $\left\langle G \mid H_{P} \cup H_{S} \cup S_{1}\right\rangle \stackrel{H_{P} \backslash H_{S}}{\longmapsto \mathcal{G}}\left\langle G^{\prime} \mid H_{P} \cup S_{1}^{\prime}\right\rangle$

Lemma 3 can be straight-forwardly extended to multiple derivation steps. This is stated in Lemma 4

Lemma 4 (Isolation of Transitive Goal-based Derivations)

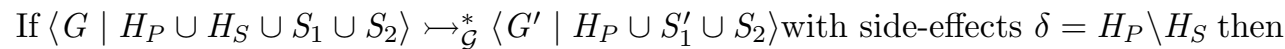
$\left\langle G \mid H_{P} \cup H_{S} \cup S_{1}\right\rangle \longmapsto_{\mathcal{G}}^{*}\left\langle G^{\prime} \mid H_{P} \cup S_{1}^{\prime}\right\rangle$

The next states that any concurrent derivation starting from a reachable CHR state can be replicated by a sequence of sequential goal-based derivations. Lemma 5 is the first step to prove the correspondence of concurrent goal-based derivations.

Lemma 5 (Sequential Reachability of Concurrent Derivation Steps)

For any sequentially reachable CHR state $\sigma$, CHR state $\sigma^{\prime}$ and rewriting side-effects $\delta$ if $\sigma \stackrel{\delta}{\longmapsto} \| \mathcal{G} \sigma^{\prime}$ then $\sigma^{\prime}$ is sequentially reachable, $\sigma \longmapsto_{\mathcal{G}}^{*} \sigma^{\prime}$ with side-effects $\delta$.

Proof

(Sketch) Via Lemma 1, we can always reduce $k$ mutually non-overlapping concurrent derivations into several applications of the (Goal Concurrency) step. Hence we can prove Lemma 5 by structural induction over the concurrent goal-based derivation steps (Lift) and (Goal Concurrency) where we use Lemmas 2 and 4 to show that concurrent derivations can always be replicated by a sequence of sequential goal-based derivations.

Theorem 3 (Sequential Reachability of Concurrent Derivations)

For any initial CHR state $\sigma$, CHR state $\sigma^{\prime}$ and CHR Program $\mathcal{P}$, if $\sigma \longmapsto{ }_{\| \mathcal{G}}^{*} \sigma^{\prime}$ then $\sigma \longmapsto_{\mathcal{G}}^{*} \sigma^{\prime}$

The above follows directly from Lemma 5 by converting each single step concurrent derivation into a sequence of sequential derivations, and showing their composibility.

From Theorem 2 and 3, we have the following corollary, which states the correspondence between concurrent goal-based $\mathrm{CHR}$ derivations and abstract $\mathrm{CHR}$ derivations.

Corollary 1 (Correspondence of Concurrent Derivations)

For any reachable CHR state $\langle G \mid S n\rangle$, CHR state $\left\langle G^{\prime} \mid S n^{\prime}\right\rangle$ and CHR program $\mathcal{P}$,

$$
\begin{array}{ll}
\text { if } & \langle G \mid S n\rangle \longmapsto{ }_{\| \mathcal{G}}^{*}\left\langle G^{\prime} \mid S n^{\prime}\right\rangle \\
\text { then } & (\operatorname{NoIds}(G) \uplus \operatorname{DropIds}(\operatorname{Sn}))=\left(\operatorname{NoIds}\left(G^{\prime}\right) \uplus \operatorname{DropIds}\left(\operatorname{Sn}^{\prime}\right)\right) \quad \vee \\
& (\operatorname{NoIds}(G) \uplus \operatorname{DropIds}(S n)) \longmapsto_{\mathcal{A}}^{*}\left(\operatorname{NoIds}\left(G^{\prime}\right) \uplus \operatorname{DropIds}\left(S n^{\prime}\right)\right)
\end{array}
$$

where $\operatorname{NoIds}=\{c \mid c \in G, c$ is a CHR constraint $\} \uplus\{e \mid e \in G, e$ is an equation $\}$ 


\subsubsection{Correspondence of Termination}

We show that all derivations from an initial state to final states in the concurrent goal-based semantics correspond to some derivation from a store to a final store in the abstract semantics. We first define rule head instances:

Definition 4.6 (Rule head instances)

For any CHR state $\sigma=\langle G, S n\rangle$ and $\mathrm{CHR}$ program $\mathcal{P}$, any $\left(H_{P} \cup H_{S}\right) \subseteq S n$ is known as a rule head instance of $\sigma$, if and only if $\exists\left(r @ H_{P}^{\prime} \backslash H_{P}^{\prime} \Longleftrightarrow t_{g} \mid B\right) \in \mathcal{P}, \exists \phi$ $\operatorname{Eqs}(S n) \models \phi \wedge t_{g}$ and $\phi\left(H_{P}^{\prime} \uplus H_{S}^{\prime}\right)=\operatorname{DropIds}\left(H_{P} \cup H_{S}\right)$.

Definition 4.7 (Active rule head instances)

For any CHR state $\sigma=\langle G, S n\rangle$ and CHR program $\mathcal{P}$, a rule head instance $H$ of $\sigma$ is said to be active if and only if there exists at least one $c \# i \in G$ such that $c \# i \in H$.

Rule head instances (Definition 4.6) are basically minimal subsets of the store which matches a rule head. Active rule head instance (Definition 4.7) additional have at least one of it is numbered constraint $c \# i$ in the goals as well. Therefore, by the definition of the goal-based semantics, active rule head instances will eventually be triggered by either the (Simplify) or (Propagate) derivation steps.

Lemma 6 (Rule instances in reachable states are always active)

For any reachable CHR state $\langle G \mid S n\rangle$, any rule head instance $H \subseteq S n$ must be active. i.e. $\exists c \# i \in H$ such that $c \# i \in G$.

Lemma 6 shows that all rule head instances in reachable states are always active. This means that by applying the semantics steps in any way, we must eventually apply the rule head instances as long as all it is constraints remain in the store.

Theorem 4 states that termination of a concurrent goal-based derivation corresponds to termination in the abstract semantics. This is of course, provided that the CHR program is terminating.

Theorem 4 (Correspondence of Termination)

For any initial CHR state $\langle G,\{\}\rangle$, final CHR state $\langle\{\}, S n\rangle$ and terminating CHR program $\mathcal{P}$,

$$
\begin{aligned}
& \text { if }\langle G \mid\{\}\rangle \longmapsto_{\| \mathcal{G}}^{*}\langle\{\} \mid S n\rangle \\
& \text { then } G \longmapsto{ }_{\mathcal{A}}^{*} \operatorname{DropIds}(S n) \text { and } \operatorname{Final}_{\mathcal{A}}(\operatorname{DropIds}(S n))
\end{aligned}
$$

We prove this theorem by first using Theorem 3 which guarantees that a concurrent goal-based derivation from an initial state to a final state corresponds to some abstract semantics derivation. We next show that final states correspond to final stores in the abstract semantics. This is done by contradiction, showing that assuming otherwise contradicts with Lemma 6 . 


\subsection{Concurrent CHR Optimizations}

In the sequential setting, there exist a wealth of optimizations (Duck 2005, Schrijvers 2005, Sneyers et al. 2005) to speed up the execution of CHR. Fortunately, many of these methods are still largely applicable to our concurrent goal-based variant as we discuss in the following. For the remainder, we assume that each goal (thread) tries the CHR rules from top-to-bottom to match the rule execution order assumed in (Duck 2005, Schrijvers 2005; Sneyers et al. 2005).

Basic constraint indexing like lookups via hashtables are still applicable with minor adaptations. For instance, the underlying hashtable implementation must be thread safe. Consider the following example:

$$
r 0 @ A(x, y), B(x), C(y) \Longleftrightarrow x>y \mid D(x, y)
$$

Suppose we have the active constraint $A(1,2) \# n$. To search for a partner constraint of the form $B(1) \# m$ and $C(2) \# p$, standard CHR compilation techniques would optimize with indexing (hashtables) which allows constant time lookup for these constraints. The use of such indexing techniques is clearly applicable in a concurrent goal execution setting as long as concurrent access of the indexing data structures are handled properly. For example, we can possibly have a concurrent active constraint $A(1,3) \# q$ which will compete with $A(1,2) \# n$ for a matching partner $B(1) \# m$. As such, hashtable implementations that facilitate such indexing must be able to be accessed and modified concurrently.

Guard optimizations/simplifications aim at simplifying guard constraints by replacing guard conditions with equivalent but simplified forms. Since guards are purely declarative, they are not influenced by concurrently executing goal threads (i.e. CHR rules). Hence, all existing guard optimizations carry over to the concurrent setting.

The join order of a CHR rule determines the order in which partner constraints are searched to execute a rule. The standard CHR optimization known as optimal join-ordering and early guard scheduling (Duck 2005) aims at executing goals with the most optimal order of partner constraints lookup and guard testing. By optimal, we refer to maximizing the use of constant time index lookup. Considering the same CHR rule $(r 0)$ above, given the active constraint $B(x)$, an optimal join-ordering is to lookup for $A(x, y)$, schedule guard $x>y$, then lookup for $C(y)$. Since our concurrent semantics does not restrict the order in which partner constraints are matched, optimal join ordering and early guard scheduling are still applicable.

Another set of optimizations tries to minimize the search for partner constraints by skipping definitely failing searches. Consider the following example:

$$
\begin{gathered}
r 1 @ A \Longleftrightarrow \cdots \\
r 2 @ A, B \Longleftrightarrow \cdots
\end{gathered}
$$

If the active goal $A$ cannot fire rule (r1) then we cannot fire rule (r2) either. Hence, after failing to fire rule (r1) we can drop goal $A$. Thus, we optimize away some definitely failing search. This statement is immediately true in the sequential setting where no other thread affects the constraint store. The situation is different in a concurrent setting where some other thread may have added in between the missing 
constraint $A$. Then, even after failing to fire (r1) we could fire rule (r2). However, we can argue that the optimization is still valid for this example. We will not violate the important condition to execute CHR rules exhaustively because the newly added constraint $A$ will eventually be executed by a goal thread which then fires rule (r1). Hence, the only concern is here that the optimization leads to indeterminism in the execution order of CHR rules which is anyway unavoidable in a concurrent setting.

Yet there are existing optimizations which are not applicable in the concurrent setting. For example, continuation optimizations (Duck 2005, Schrijvers 2005) are not entirely applicable. Consider the following CHR rule:

$$
r 4 @ A(x), A(y) \Longleftrightarrow x==y \mid \cdots
$$

Given an active constraint $A(1) \# n$, fail continuation optimization will infer that if we fail to fire the rule with $A(1) \# n$ matching $A(x)$, there is no point trying to match it with $A(y)$ because it will most certainly fail as well, assuming that the store never changes. In a concurrent goal execution setting, we cannot assume that the store never changes (while trying to execute a CHR). For instance, after failing to trigger the rule by matching $A(1) \# n$ with $A(x)$, suppose that a new active goal $A(1) \# m$ is added to the store concurrently. Now when we match $A(1) \# n$ to $A(y)$ we can find match the partner $A(1) \# m$ with $A(x)$, hence breaking the assumptions of the fail continuation optimization.

Late (also known as delayed) storage optimization (Duck 2005) aims at delaying the storage of a goal $g$, until the latest point of its execution where $g$ is possibly a partner constraint of another active constraint. Consider the following example:

$$
\begin{aligned}
& r 1 @ P_{1} \Longrightarrow Q \\
& r 2 @ P_{2}, T_{1} \Longleftrightarrow R \\
& r 3 @ P_{3}, R_{1} \Longleftrightarrow \text { True } \\
& r 4 @ P_{4} \Longrightarrow S \\
& r 5 @ P_{5}, S_{1} \Longleftrightarrow \text { True }
\end{aligned}
$$

Note to distinguish the rule heads, we annotate each rule head with a subscript integer (eg. $\left.P_{x}\right)$. With late storage analysis techniques described in (Duck 2005), we can delay storage of an active constraint $P$ until just before the execution of the body of $r 4$. This is because the execution of goal $S$ (obtained from firing of $r 4$ ) can possibly trigger $r 5$. While this is safe in the sequential goal execution scheme, it is possible that rule matches are missing in the concurrent goal execution setting. Consider the case where we have some simultaneously active goals $P \# n$ and $T \# m$. Since $P \# n$ is only stored when its execution has reached $r 4$, the match $r 2$ can be missed entirely by both active parallel goals $P \# n$ and $T \# m$. Specifically, this happens if goal $T \# m$ is activated only after $P \# n$ has tried matching with $P_{2}$ (of $r 2$ ), but completes goal execution (by trying $T_{1}$ of $r 2$, and failing to match) before goal $P \# n$ is stored. Hence, we conclude that we cannot safely implement late storage in the concurrent setting.

\section{Related Work}

We review prior work on execution schemes for CHR and production rule systems. 


\subsection{CHR Execution Schemes}

There exists a wealth of prior work on the semantics of CHR. We refer to (Sneyers et al. ) for a comprehensive summary. Our focus here is on the operational CHR semantics and we briefly review the most relevant works.

The theoretical (a.k.a. high-level) operational semantics (Frühwirth 1998) is derived from the abstract semantics and inherits its high degree of indeterminism. The theoretical semantics has been mainly used for the study of high-level properties such as confluence (Abdennadher 1997, Abdennadher et al. 1999). Confluence analysis has been exploited to study the degree of concurrency in CHR programs (Frühwirth 2005, Schrijvers and Sulzmann 2008). None of these works however provide direct glues how to systematically execute concurrent programs.

In (Duck et al. 2004, De Koninck et al. 2008, De Koninck et al. 2008) some systematic, highly deterministic semantics have been developed to achieve efficient implementation schemes. However, these semantics are inherently single-threaded. Our motivation is to obtain systematic yet concurrent semantics which led us to develop the goal-based concurrent semantics presented in this paper. In the special case of a single goal thread, our semantics is equivalent to the refined operational semantics given in (Duck et al. 2004; De Koninck et al. 2008, De Koninck et al. 2008).

There are only few works which explore different semantics, other than the theoretical or abstract semantics, to address concurrency. The work in (Sarna-Starosta and Ramakrishnan 2007) adopts a set-based semantics and supports tabled, possible concurrent, rule execution. This execution scheme is not applicable to CHR programs in general which usually assume a multi-set based semantics. The recent work in (Betz et al. 2009) takes a new stab at concurrency by introducing the notion of persistent constraints. The idea is to split the store into linear (multi-set like) and persistent (set like) constraints. We are not aware of any evidence which shows that this approach supports effective concurrency in practice. Our approach leads to an efficient parallel implementation as we explain in the next section.

\subsection{From Concurrent to Parallel CHR Execution}

In our earlier works (Lam and Sulzmann 2007, Sulzmann and Lam 2008) we have developed a parallel CHR implementation scheme based on an informally described concurrent goal-based execution scheme, see Section 3 in (Sulzmann and Lam 2008). The present works provides a concise formal treatment of the implemented concurrent goal-based execution scheme. In our implementation, multiple threads, each executing a unique CHR goal, are executed in parallel on multiple processor cores. Parallel goal executions are largely asynchronous, only implicitly synchronizing via the shared constraint store. Atomic CHR execution is guaranteed via advanced synchronization primitives such as Software Transactional Memory. We refer to (Sulzmann and Lam 2008) for a thorough description of the more subtle implementation details. Our experimental results reported in (Sulzmann and Lam 2008) show that we achieve good scalability when the number of processor cores increases. The overhead of the parallel implementation is fairly minor compared to a single- 


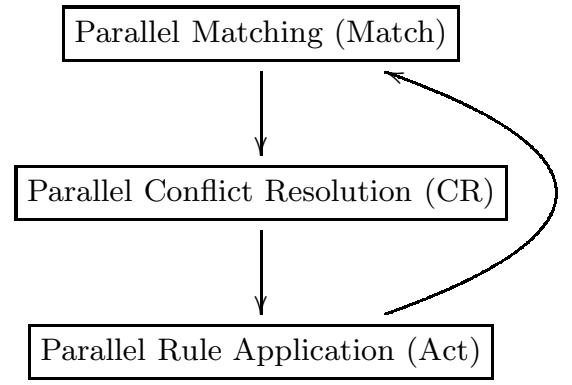

Fig. 8. Parallel Production Rule Execution Cycles

threaded implementations thanks to the use of lock-free algorithms. Optimization methods applicable in the concurrent/parallel setting are discussed in the earlier Section 4.3 .

\subsection{Parallel Production Rule Systems}

Parallel execution models of forward chaining production rule based languages (e.g. OPS5 (Forgy and McDermott 1977)) have been widely studied in the context of production rule systems. A production rule system is defined by a set of multiheaded production rules (analogous to CHR rules) and a set of assertions (analogous to the CHR store). Production rule systems are richer than the CHR language, consisting of user definable execution strategies and negated rule heads. This makes parallelizing production rule execution extremely difficult, because rule application is not monotonic (rules may not be applied in a larger context). As such, many previous works in parallel production rule systems focuses on efficient means of maintaining correctness of parallel rule execution (e.g. data dependency analysis (Ishida 1991), sequential to parallel program transformation (Gamble 1990)), with respect to such user specified execution strategies. These works can be classified under two approaches, namely synchronous and asynchronous parallel production systems.

For synchronous parallel production systems (e.g. UMPOPS (Gupta et al. 1988)), multiple processors/threads run in parallel. They are synchronized by execution cycles of the production systems. Figure 8 illustrates the production cycle of a typical production rule system, consisting of three execution phases. In the (Match) phase, all rule matches are computed. Conflict resolution (CR) involves filtering out matches that do not conform to the user specified rule execution strategy, while (Act) applies the rule matches that remains (known as the eligible set) after the $(\mathrm{CR})$ phase. By synchronizing parallel rule execution in production cycles, a larger class of user specified execution strategies can be supported since execution is staged.

Matching in synchronous production rule systems often use some variant of the RETE network (Forgy 1982). RETE is an incremental matching algorithm where 
$r 1 @ A(x) \backslash B(x), C(y) \Longleftrightarrow D(x, y) \quad\{A(1), A(2), B(1), B(2), C(3)\}$

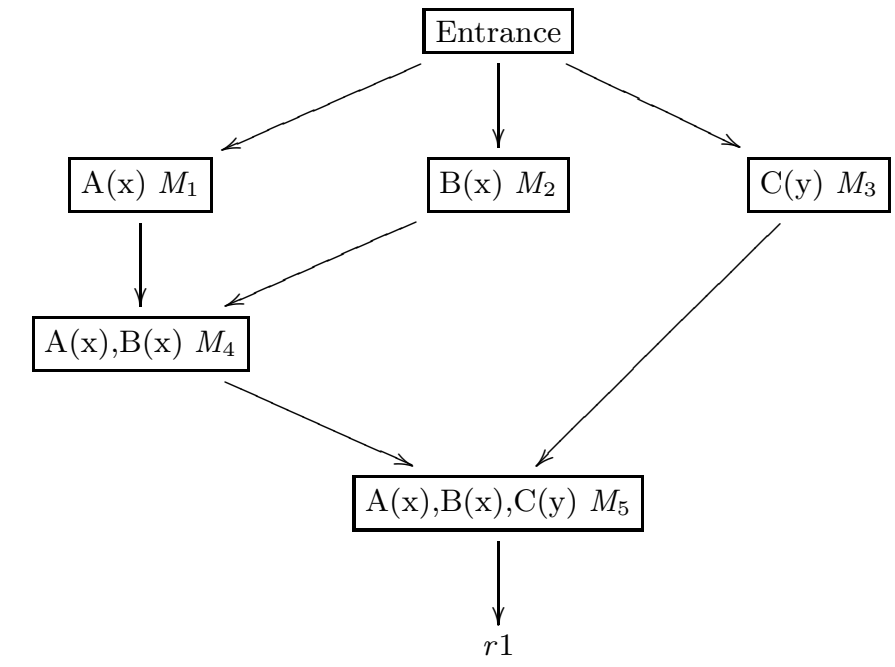

$M_{1}=\{A(1), A(2)\} \quad M_{2}=\{B(1), B(2)\} \quad M_{3}=\{C(3)\}$

$M_{4}=\{\{A(1), B(1)\},\{A(2), B(2)\}\}$

$M_{5}=\{\{A(1), B(1), C(3)\},\{A(2), B(2), C(3)\}\}$

Fig. 9. Example of a RETE network, in CHR context

matching is done eagerly (data driven) in that each newly added assertion (constraint in CHR context) triggers computation of all it is possible matches to rule heads. Figure 9 illustrates a RETE network (acyclic graph), described in CHR context. Root node is the entrance where new constraints are added. Intermediate nodes with single output edges are known as alpha nodes. Intermediate nodes with two output edges are beta nodes, representing joins between alpha nodes. Each alpha node is associated with a set of constraint matching its pattern, while a beta node is associated with a set of partial/complete matches. Parallel implementation of RETE (Mahajan and Kumar 1990) allows distinct parts of the network to be computed in parallel.

The most distinct characteristic of RETE is that partial matches are computed and stored. This and the eager nature of RETE matching is suitable for production rule systems as assertions (constraints) are propagated (not deleted) by default. Hence computing all matches rarely results in redundancy. Traditional CHR systems do not advocate this eager matching scheme because doing so results to many redundancies, due to overlapping simplified matching heads. Eager matching algorithms is also proved in (D.P. Miranker and Gadbois 1990) to have a larger asymptotic worst-case space complexity than lazy matching algorithms.

In (Miranker 1990), the matching algorithm TREAT is proposed. TREAT is similar to RETE, except it does not store partial matches. TREAT performs better 
than RETE if the overhead of maintaining and storing partial matches outweighs that of re-computing partial matches.

Asynchronous parallel production rule systems (e.g. Swarm (Gamble 1990), CREL (Miranker et al. 1989)) introduce parallel rule execution via asynchronously running processors/threads. In such systems, rules can fire asynchronously (not synchronized by production cycles), hence enforcing execution strategies is more difficult and limited. Similar to implementations of goal based CHR semantics rule matching is such systems often use a variant of the LEAPS (D.P. Miranker and Gadbois 1990) lazy matching algorithm.

\subsubsection{Observations}

Staging executions in synchronous parallel production rule systems allows for flexibility in imposing execution strategies, but at a cost. In (Neiman 1991), synchronous execution of UMPOPS production rule system is shown to be less efficient than asynchronous execution. Hence it is clear that synchronous systems will only be necessary if we wish to impose some form of execution strategies on top of the abstract CHR semantics (e.g. rule-priority, refined operational semantics). We are interested in concurrent CHR semantics on the abstract CHR semantics. Its nondeterminism and monotonicity property provides us with the flexibility to avoid executing threads in strict staging cycles. Thus our approach is very similar to asynchronous parallel production rule systems.

Lazy matching in single-threaded CHR execution is the best choice, since we only ever have one thread of execution and wish to avoid computing overlapping (redundant) rule head matches. No doubt that in a parallel setting, eager matching (like RETE, TREAT) may be more optimal if the executed CHR program consist of rules with more propagated heads. This is because we compute more matches in parallel with brute force (find all match) parallelism and we can get away with less redundancy. Yet to cater for the general case (more simplified heads), we again choose lazy matching.

We therefore conclude that the goal-based execution model of CHR is still the ideal choice for a parallel implementation of the abstract CHR semantics. For CHR with rule priorities or refined CHR operational semantics, a variant of the synchronous parallel production rule execution is a possible choice. We leave this topic for future work.

\section{Conclusion}

We have introduced a novel concurrent goal-based CHR semantics which is inspired by traditional single-threaded (sequential) goal-based CHR execution models. Existing CHR semantics aim at introducing specific execution strategies (e.g. ordered goal execution, rule priorities) on top of the CHR abstract semantics, hence adding more determinism. In contrast, the concurrent goal-based CHR semantics exploits the inherent non-deterministic and concurrent abstract CHR semantics, while introducing a systematic goal-based execution strategy. We have shown that all concur- 
rent derivations can be replicated in the sequential goal-based semantics and that there is a correspondence between the sequential goal-based semantics and the abstract CHR semantics. Thus, establishing correctness of our concurrent goal-based CHR semantics. Our semantics provides the basis for an efficient parallel CHR implementation. The details are studied elsewhere (Sulzmann and Lam 2008).

An interesting question is how our concurrent semantics can help to parallelize an existing single-threaded semantics such as (Duck et al. 2004). We leave the study of this issue for future work.

\section{Acknowledgments}

We thank the reviewers for their helpful comments on a previous version of this paper.

\section{References}

AbDennadher, S. 1997. Operational semantics and confluence of constraint propagation rules. In Proc. of CP'97. LNCS. Springer-Verlag, 252-266.

Abdennadher, S., Fruhwirth, T., And Meuss, H. 1999. Confluence and semantics of constraint simplification rules. Constraints Journal 4.

Betz, H., Raiser, F., And Frühwirth, T. 2009. Persistent constraints in Constraint Handling Rules. In WLP '09: Proc. 23rd Workshop on (Constraint) Logic Programming. To appear.

De Koninck, L., Stuckey, P., And Duck, G. 2008. Optimizing compilation of CHR with rule priorities. In Proc. of FLOPS'08. LNCS, vol. 4989. Springer-Verlag, 32-47.

D.P. Miranker, D. Brant, B. L. And Gadbois, D. 1990. On the performance of lazy matching in production systems. In In proceedings of International Conference on Artificial Intelligence AAAI. 685-692.

Duck, G. J. 2005. Compilation of Constraint Handling Rules. Ph.D. thesis, The University of Melbourne.

Duck, G. J., Stuckey, P. J., de la Banda, M. J. G., And Holzbaur, C. 2004. The refined operational semantics of Constraint Handling Rules. In Proc of ICLP'04. LNCS, vol. 3132. Springer-Verlag, 90-104.

FORGY, C. 1982. Rete: A fast algorithm for the many patterns/many objects match problem. Artif. Intell. 19, 1, 17-37.

Forgy, C. AND MCDermott, J. P. 1977. Ops, a domain-independent production system language. In IJCAI. 933-939.

FrüHwirth, T. 1998. Theory and practice of constraint handling rules. Journal of Logic Programming, Special Issue on Constraint Logic Programming 37, 1-3, 95-138.

Frühwirth, T. 2005. Parallelizing union-find in Constraint Handling Rules using confluence analysis. In Proc. of ICLP'05. LNCS, vol. 3668. Springer-Verlag, 113-127.

Frühwirth, T. 2006. Constraint handling rules: the story so far. In Proc. of PPDP '06. ACM Press, 13-14.

Gamble, R. F. 1990. Transforming rule-based programs: from the sequential to the parallel. In IEA/AIE '90: Proceedings of the 3rd international conference on Industrial and engineering applications of artificial intelligence and expert systems. ACM, New York, NY, USA, 854-863. 
Sequential Goal-based Semantics $k$-closure

$$
\text { (k-Step) } \quad \sigma \longmapsto_{\mathcal{G}}^{0} \sigma \quad \frac{\sigma \stackrel{\delta}{\mathcal{G}}^{\prime} \sigma^{\prime} \longmapsto_{\mathcal{G}}^{k} \sigma^{\prime \prime}}{\sigma \longmapsto_{\mathcal{G}}^{k+1} \sigma^{\prime \prime}}
$$

Concurrent Goal-based Semantics $k$-closure

$$
\text { (k-Step) } \quad \sigma \longmapsto \|_{\| \mathcal{G}}^{0} \sigma
$$

$$
\frac{\stackrel{\delta}{\|} \| \mathcal{G} \sigma^{\prime} \quad \sigma^{\prime} \stackrel{k}{\| \mathcal{G}}^{\prime \prime} \sigma^{\prime \prime}}{\sigma \longmapsto{ }_{\| \mathcal{G}}^{k+1} \sigma^{\prime \prime}}
$$

Fig. 10. $k$-closure derivation steps

Gupta, A., Forgy, C., Kalp, D., Newell, A., And Tambe, M. 1988. Parallel ops5 on the encore multimax. In ICPP (1). 71-280.

IshidA, T. 1991. Parallel rule firing in production systems. IEEE Transactions on Knowledge and Data Engineering 3, 1, 11-17.

Lam, E. S. L. And Sulzmann, M. 2007. A concurrent Constraint Handling Rules implementation in Haskell with software transactional memory. In Proc. of ACM SIGPLAN Workshop on Declarative Aspects of Multicore Programming (DAMP'07). 19-24.

Mahajan, M. And Kumar, V. K. P. 1990. Efficient parallel implementation of rete pattern matching. Comput. Syst. Sci. Eng. 5, 3, 187-192.

Miranker, D. P. 1990. TREAT: a new and efficient match algorithm for AI production systems. Morgan Kaufmann Publishers Inc., San Francisco, CA, USA.

Miranker, D. P., Kuo, C., And Browne, J. C. 1989. Parallelizing transformations for a concurrent rule execution language. Tech. rep., Austin, TX, USA.

Neiman, D. E. 1991. Control issues in parallel rule-firing production systems. In in Proceedings of National Conference on Artificial Intelligence. 310-316.

Sarna-Starosta, B. and Ramakrishnan, C. R. 2007. Compiling constraint handling rules for efficient tabled evaluation. In Proc. of PADL'07. LNCS, vol. 4354. Springer, $170-184$.

SChriJvers, T. 2005. Analyses, optimizations and extensions of Constraint Handling Rules: Ph.D. summary. In Proc. of ICLP'05. LNCS, vol. 3668. Springer-Verlag, 435436.

Schrijvers, T. And Sulzmann, M. 2008. Transactions in constraint handling rules. In Proc. of ICLP'08. LNCS, vol. 5366. Springer, 516-530.

Sneyers, J., Schrijvers, T., And Demoen, B. 2005. Guard and continuation optimization for occurrence representations of chr. In Proc. of ICLP'05. LNCS, vol. 3668. Springer-Verlag, 83-97.

Sneyers, J., Van Weert, P., Schrijvers, T., And De Koninck, L. As time goes by: Constraint handling rules - a survey of chr research from 1998 to 2007. To appear in TPLP.

Sulzmann, M. And Lam, E. S. L. 2008. Parallel execution of multi-set constraint rewrite rules. In Proc. of PPDP'08. ACM Press, 20-31. 


\section{Proofs}

In this section, we provide the proofs of the Lemmas and Theorems discussed in this paper. Because many of our proofs rely on inductive steps on the derivations, we define $k$-step derivations to facilitate the proof mechanisms. Figure 10 shows $k$-step derivations of the sequential goal-based derivations $\stackrel{\delta}{\longmapsto} \mathcal{G}$ and the concurrent goal-based derivations $\stackrel{\delta}{\longmapsto} \| \mathcal{G}$.

\subsubsection{Proof of Correspondence of Derivations}

Theorem 2 (Correspondence of Sequential Derivations) For any reachable CHR state $\langle G \mid S n\rangle$, CHR state $\left\langle G^{\prime} \mid S n^{\prime}\right\rangle$ and CHR Program $\mathcal{P}$,

$$
\begin{array}{ll}
\text { if } & \langle G \mid S n\rangle \longmapsto_{\mathcal{G}}^{*}\left\langle G^{\prime} \mid S n^{\prime}\right\rangle \\
\text { then } & (\operatorname{NoIds}(G) \uplus \operatorname{DropIds}(\operatorname{Sn}))=\left(\operatorname{NoIds}\left(G^{\prime}\right) \uplus \operatorname{DropIds}\left(\operatorname{Sn}^{\prime}\right)\right) \quad \vee \\
& (\operatorname{NoIds}(G) \uplus \operatorname{DropIds}(\operatorname{Sn})) \longmapsto_{\mathcal{A}}^{*}\left(\operatorname{NoIds}\left(G^{\prime}\right) \uplus \operatorname{DropIds}\left(\operatorname{Sn}^{\prime}\right)\right)
\end{array}
$$

where NoIds $=\{c \mid c \in G, c$ is a CHR constraint $\} \uplus\{e \mid e \in G, e$ is an equation $\}$

Proof

We prove that for all finite $n$ and reachable states $\langle G \mid S n\rangle,\left\langle G^{\prime} \mid S n^{\prime}\right\rangle,\langle G \mid S n\rangle \longmapsto_{\mathcal{G}}^{n}$ $\left\langle G^{\prime} \mid S n^{\prime}\right\rangle$ either yields equivalent abstract stores or corresponds to some abstract semantics derivation. We prove by induction on the derivation steps $n$. Showing that goal-based derivation of any finite $n$ steps satisfying one of the following conditions:

- $(\mathbf{C 1})(\operatorname{NoIds}(G) \uplus \operatorname{DropIds}(S n))=\left(\operatorname{NoIds}\left(G^{\prime}\right) \uplus \operatorname{DropIds}\left(S n^{\prime}\right)\right)$

- (C2) $(\operatorname{NoIds}(G) \uplus \operatorname{DropIds}(S n)) \longmapsto_{\mathcal{A}}^{*}\left(\operatorname{NoIds}\left(G^{\prime}\right) \uplus \operatorname{DropIds}\left(\operatorname{Sn}^{\prime}\right)\right)$

We have the following axioms, by definition of the functions NoIds and DropIds, for any goals $G$ or store $S n$ :

- (a1) For any equation $e, \operatorname{NoIds}(\{e\} \uplus G)=\{e\} \uplus \operatorname{NoIds}(G)$

- (a2) For any equation $e, \operatorname{DropIds}(\{e\} \cup S n)=\{e\} \uplus \operatorname{DropIds}(S n)$

- (a3) For any numbered constraint $c \# i, \operatorname{NoIds}(\{c \# i\} \uplus G)=\operatorname{NoIds}(G)$

- (a4) For any numbered constraint $c \# i$, DropIds $(\{c \# i\} \cup S n)=\{c\} \uplus \operatorname{DropIds}(\operatorname{Sn})$

- (a5) For any CHR constraint $c, N o I d s(\{c\} \uplus G)=\{c\} \uplus \operatorname{NoIds}(G)$

- (a6) For any store $S n^{\prime}, \operatorname{DropIds}\left(S n \cup S n^{\prime}\right)=\operatorname{DropIds}(S n) \uplus \operatorname{DropIds}\left(S n^{\prime}\right)$

(a1) and (a2) are so because NoIds and DropIds have no effect on equations. (a3) is true because NoIds is defined to drop numbered constraints. (a4) is true because DropIds is defined to remove identifier components of numbered constraints. We have (a5) because NoIds has no effect on CHR constraints. By definition of DropIds, (a6) is true.

Base case: We consider $\langle G \mid S n\rangle \hookrightarrow_{\mathcal{G}}^{0}\left\langle G^{\prime} \mid S n^{\prime}\right\rangle$. By definition of $\longmapsto_{\mathcal{G}}^{0}$, we have $G=G^{\prime}$ and $S n=S n^{\prime}$. Hence $(\operatorname{NoIds}(G) \uplus \operatorname{DropIds}(S n))=\left(\operatorname{NoIds}\left(G^{\prime}\right) \uplus\right.$ $\left.\operatorname{DropIds}\left(S n^{\prime}\right)\right)$ and we are done.

Inductive case: We assume that the theorem is true for some finite $k>0$, hence

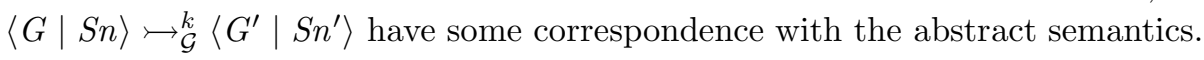


We now prove that by extending these $k$ derivations with another step, we preserve correspondence, namely $\langle G \mid S n\rangle \longmapsto_{\mathcal{G}}^{k}\left\langle G^{\prime} \mid S n^{\prime}\right\rangle \stackrel{\delta}{\longmapsto_{\mathcal{G}}}\left\langle G^{\prime \prime} \mid S n^{\prime \prime}\right\rangle$ has a correspondence with the abstract semantics. We prove this by considering all possible form of derivation step, step $k+1$ can take:

- (Solve) $k+1$ step is of the form $\left\langle\{e\} \uplus G^{\prime \prime \prime} \mid S n^{\prime}\right\rangle \stackrel{\delta}{\longmapsto} \mathcal{G}\left\langle W \uplus G^{\prime \prime \prime} \mid\{e\} \cup S n^{\prime}\right\rangle$ such that for some $G^{\prime \prime \prime}$ and $W$

$$
G^{\prime}=\{e\} \uplus G^{\prime \prime \prime}, G^{\prime \prime}=W \uplus G^{\prime \prime \prime} \text { and } S n^{\prime \prime}=\{e\} \cup S n^{\prime} \quad(\text { a solve })
$$

where $e$ is an equation, $W=W a k e U p(e, S n)$ contains only goals of the form $c \# i$. This is because (Solve) only wakes up stored numbered constraints. Hence,

$$
\begin{aligned}
\operatorname{NoIds}\left(G^{\prime \prime}\right) \uplus \operatorname{DropIds}\left(S n^{\prime \prime}\right) & =\operatorname{NoIds}\left(W \uplus G^{\prime \prime \prime}\right) \uplus \operatorname{DropIds}\left(\{e\} \cup \operatorname{Sn} n^{\prime}\right)\left(\mathbf{a}_{\text {solve }}\right) \\
& =\operatorname{NoIds}\left(G^{\prime \prime \prime}\right) \uplus \operatorname{DropIds}\left(\{e\} \cup S n^{\prime}\right)(\mathbf{a 3}) \\
& =\operatorname{NoIds}\left(G^{\prime \prime \prime}\right) \uplus\{e\} \uplus \operatorname{DropIds}\left(\operatorname{Sn}^{\prime}\right)(\mathbf{a 2}) \\
& =\operatorname{NoIds}\left(\{e\} \uplus G^{\prime \prime \prime}\right) \uplus \operatorname{DropIds}\left(\operatorname{Sn}^{\prime}\right)(\mathbf{a} 1) \\
& =\operatorname{NoIds}\left(G^{\prime}\right) \uplus \operatorname{DropIds}\left(\operatorname{Sn}^{\prime}\right)\left(\mathbf{a}_{\text {solve }}\right)
\end{aligned}
$$

Hence we can conclude that the evaluated store of derivation step $k+1$ is equivalent to abstract store of evaluated store of step $k$, therefore satisfying condition (C1).

- (Activate) $k+1$ step is of the form $\left\langle\{c\} \uplus G^{\prime \prime \prime} \mid S n^{\prime}\right\rangle \stackrel{\delta}{\longmapsto} \mathcal{G}\left\langle\{c \# i\} \uplus G^{\prime \prime \prime} \mid\{c \# i\} \cup S n^{\prime}\right\rangle$ such that for some $G^{\prime \prime \prime}$

$$
G^{\prime}=\{c\} \uplus G^{\prime \prime \prime}, G^{\prime \prime}=\{c \# i\} \uplus G^{\prime \prime \prime} \text { and } S n^{\prime \prime}=\{c \# i\} \cup S n^{\prime} \quad\left(\mathbf{a}_{\text {act }}\right)
$$

Hence,

$$
\begin{array}{rlrl}
\operatorname{NoIds}\left(G^{\prime \prime}\right) \uplus \operatorname{DropIds}\left(S n^{\prime \prime}\right) & & \\
& =\operatorname{NoIds}\left(\{c \# i\} \uplus G^{\prime \prime \prime}\right) \uplus \operatorname{DropIds}\left(\{c \# i\} \cup S n^{\prime}\right) & & \left(\mathbf{a}_{\text {act }}\right) \\
& =\operatorname{NoIds}\left(G^{\prime \prime \prime}\right) \uplus \operatorname{DropIds}\left(\{c \# i\} \cup S n^{\prime}\right) & & \text { (a3) } \\
& =\operatorname{NoIds}\left(G^{\prime \prime \prime}\right) \uplus\{c\} \uplus \operatorname{DropIds}\left(S n^{\prime}\right) & \text { (a4) } \\
& =\operatorname{NoIds}\left(\{c\} \uplus G^{\prime \prime \prime}\right) \uplus \operatorname{DropIds}\left(S n^{\prime}\right) & & \text { (a5) } \\
& =\operatorname{NoIds}\left(G^{\prime}\right) \uplus \operatorname{DropIds}\left(S n^{\prime}\right) & & \text { (aact) }
\end{array}
$$

Hence we can conclude that evaluated store of derivation step $k+1$ is equivalent to abstract store of evaluated store of step $k$, therefore satisfying condition (C1).

- (Simplify) $k+1$ step is of the form $\left\langle\{c \# i\} \uplus G^{\prime \prime \prime} \mid H_{P} \cup\{c \# i\} \cup H_{S} \cup S n^{\prime \prime \prime}\right\rangle \stackrel{\delta}{\longmapsto}$ $\left\langle B \uplus G^{\prime \prime \prime} \mid H_{P} \cup S n^{\prime \prime \prime}\right\rangle$ for some $H_{P}, H_{S}$ and $B$ such that for some $G^{\prime \prime \prime}$ and $S n^{\prime \prime \prime}$

$$
\begin{aligned}
& S n^{\prime}=H_{P} \cup\{c \# i\} \cup H_{S} \cup S n^{\prime \prime \prime}, S n^{\prime \prime}=H_{P} \cup S n^{\prime \prime \prime}, \\
& G^{\prime}=\{c \# i\} \uplus G^{\prime \prime \prime} \text { and } G^{\prime \prime}=B \uplus G^{\prime \prime \prime}
\end{aligned}
$$

and there exists a CHR rule $r @ H_{P}^{\prime} \backslash H_{S}^{\prime} \Longleftrightarrow t_{g} \mid B^{\prime}$ such that exists $\phi$ where

$$
\begin{array}{ll}
\operatorname{DropIds}\left(\{c \# i\} \cup H_{S}\right)=\phi\left(H_{S}^{\prime}\right) & \operatorname{DropId}\left(H_{P}\right)=\phi\left(H_{P}^{\prime}\right) \\
E q\left(S n^{\prime \prime \prime}\right) \models \phi \wedge t_{g} \quad B=\phi\left(B^{\prime}\right) & (\mathbf{a 2} \text { simp })
\end{array}
$$

Hence,

$$
\begin{aligned}
\operatorname{NoId} & \left(G^{\prime}\right) \uplus \operatorname{DropIds}\left(\operatorname{Sn}^{\prime}\right) & & \\
& =\operatorname{NoIds}\left(\{c \# i\} \uplus G^{\prime \prime \prime}\right) \uplus \operatorname{DropIds}\left(H_{P} \cup\{c \# i\} \cup H_{S} \cup S^{\prime \prime \prime}\right) & & \left(\mathbf{a}_{\text {simp }}\right) \\
& =\operatorname{NoIds}\left(G^{\prime \prime \prime}\right) \uplus \operatorname{DropIds}\left(H_{P} \cup\{c \# i\} \cup H_{S} \cup \operatorname{Sn}^{\prime \prime \prime}\right) & & \left(\mathbf{a}_{\mathbf{3}}\right) \\
& =\operatorname{NoIds}\left(G^{\prime \prime \prime}\right) \uplus \operatorname{DropIds}\left(H_{P}\right) \uplus \operatorname{DropIds}\left(\{c \# i\} \cup H_{S}\right) \uplus \operatorname{DropIds}\left(\operatorname{Sn}^{\prime \prime \prime}\right) & & \left(\mathbf{a}_{\mathbf{6}}\right) \\
& =\operatorname{NoIds}\left(G^{\prime \prime \prime}\right) \uplus \phi\left(H_{P}^{\prime}\right) \uplus \phi\left(H_{S}^{\prime}\right) \uplus \operatorname{DropIds}\left(\operatorname{Sn}^{\prime \prime \prime}\right) & & \left(\mathbf{a} \mathbf{2}_{\text {simp }}\right)
\end{aligned}
$$


By definition of the abstract semantics and $a 2_{\text {simp }}$, we know that we have the rule application $\phi\left(H_{P}^{\prime}\right) \cup \phi\left(H_{S}^{\prime}\right) \longmapsto_{\mathcal{A}} \phi\left(B^{\prime}\right)$ Therefore, by monotonicity of CHR rewriting (Theorem 11)

$$
\begin{aligned}
& \operatorname{NoId}\left(G^{\prime}\right) \uplus \operatorname{DropIds}\left(S n^{\prime}\right) \\
& =\operatorname{NoIds}\left(G^{\prime \prime \prime}\right) \uplus \phi\left(H_{P}^{\prime}\right) \uplus \phi\left(H_{S}^{\prime}\right) \uplus \operatorname{DropIds}\left(S n^{\prime \prime \prime}\right)
\end{aligned}
$$

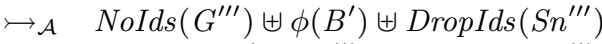

$$
\begin{aligned}
& =\operatorname{NoIds}\left(\phi\left(B^{\prime}\right) \uplus G^{\prime \prime \prime}\right) \uplus \operatorname{DropIds}\left(S n^{\prime \prime \prime}\right) \\
& =\operatorname{NoIds}\left(G^{\prime \prime}\right) \uplus \operatorname{DropIds}\left(\operatorname{Sn}^{\prime \prime}\right)
\end{aligned}
$$

Therefore, we have $\operatorname{NoId}(G) \uplus \operatorname{DropIds}(\operatorname{Sn}) \longmapsto_{\mathcal{A}}^{*} \operatorname{NoId}\left(G^{\prime}\right) \uplus \operatorname{DropIds}\left(\operatorname{Sn}^{\prime}\right) \longmapsto_{\mathcal{A}}$ $\operatorname{NoIds}\left(G^{\prime \prime}\right) \uplus \operatorname{DropIds}\left(S n^{\prime \prime}\right)$, such that the $k+1$ goal-based derivation step satisfy condition (C2).

- (Propagate) $k+1$ step is of the form $\left\langle\{c \# i\} \uplus G^{\prime \prime \prime} \mid H_{P} \cup\{c \# i\} \cup H_{S} \cup S n^{\prime \prime \prime}\right\rangle \stackrel{\delta}{\longmapsto} \mathcal{G}$ $\left\langle B \uplus\{c \# i\} \uplus G^{\prime \prime \prime} \mid H_{P} \cup\{c \# i\} \cup S n^{\prime \prime \prime}\right\rangle$ for some $H_{P}, H_{S}$ and $B$ such that for some $G^{\prime \prime \prime}$ and $S n^{\prime \prime \prime}$

$$
\begin{aligned}
& S n^{\prime}=H_{P} \cup\{c \# i\} \cup H_{S} \cup S n^{\prime \prime \prime}, S n^{\prime \prime}=H_{P} \cup\{c \# i\} \cup S n^{\prime \prime \prime}, \\
& G^{\prime}=\{c \# i\} \uplus G^{\prime \prime \prime} \text { and } G^{\prime \prime}=B \uplus\{c \# i\} \uplus G^{\prime \prime \prime}
\end{aligned}
$$

and there exists a CHR rule $r @ H_{P}^{\prime} \backslash H_{S}^{\prime} \Longleftrightarrow t_{g} \mid B^{\prime}$ such that exists $\phi$ where

$$
\begin{aligned}
& \operatorname{DropIds}\left(H_{S}\right)=\phi\left(H_{S}^{\prime}\right) \quad \operatorname{DropIds}\left(\{c \# i\} \cup H_{P}\right)=\phi\left(H_{P}^{\prime}\right) \\
& E q\left(S n^{\prime \prime \prime}\right) \models \phi \wedge t_{g} \quad B=\phi\left(B^{\prime}\right)
\end{aligned}
$$

Hence,

$$
\begin{aligned}
\operatorname{NoId} & \left(G^{\prime}\right) \uplus \operatorname{DropIds}\left(\operatorname{Sn}^{\prime}\right) & & \\
& =\operatorname{NoIds}\left(\{c \# i\} \uplus G^{\prime \prime \prime}\right) \uplus \operatorname{DropIds}\left(H_{P} \cup\{c \# i\} \cup H_{S} \cup \operatorname{Sn}^{\prime \prime \prime}\right) & & \text { (a1 } \mathbf{p r o p}) \\
& =\operatorname{NoIds}\left(G^{\prime \prime \prime}\right) \uplus \operatorname{DropIds}\left(H_{P} \cup\{c \# i\} \cup H_{S} \cup \operatorname{Sn}^{\prime \prime \prime}\right) & & \left(\mathbf{a}_{3}\right) \\
& =\operatorname{NoIds}\left(G^{\prime \prime \prime}\right) \uplus \operatorname{DropIds}\left(\{c \# i\} \cup H_{P}\right) \uplus \operatorname{DropIds}\left(H_{S}\right) \uplus \operatorname{DropIds}\left(\operatorname{Sn}^{\prime \prime \prime}\right) & & \left(\mathbf{a}_{\mathbf{6}}\right) \\
& =\operatorname{NoIds}\left(G^{\prime \prime \prime}\right) \uplus \phi\left(H_{P}^{\prime}\right) \uplus \phi\left(H_{S}^{\prime}\right) \uplus \operatorname{DropIds}\left(\operatorname{Sn}^{\prime \prime \prime}\right) & & \left(\mathbf{a} \mathbf{2}_{\text {prop }}\right)
\end{aligned}
$$

By definition of the abstract semantics and $a 2_{\text {simp }}$, we know that we have the rule application $\phi\left(H_{P}^{\prime}\right) \cup \phi\left(H_{S}^{\prime}\right) \longmapsto_{\mathcal{A}} \phi\left(B^{\prime}\right)$ Therefore, by monotonicity of CHR rewriting (Theorem 1)

$$
\begin{aligned}
& \operatorname{NoId}\left(G^{\prime}\right) \uplus \operatorname{DropIds}\left(S n^{\prime}\right) \\
& =\operatorname{NoIds}\left(G^{\prime \prime \prime}\right) \uplus \phi\left(H_{P}^{\prime}\right) \uplus \phi\left(H_{S}^{\prime}\right) \uplus \operatorname{DropIds}\left(S n^{\prime \prime \prime}\right) \\
& \longmapsto_{\mathcal{A}} \quad \operatorname{NoIds}\left(G^{\prime \prime \prime}\right) \uplus \phi\left(B^{\prime}\right) \uplus \operatorname{DropIds}\left(S^{\prime \prime \prime}\right) \\
& =\operatorname{NoIds}\left(\phi\left(B^{\prime}\right) \uplus G^{\prime \prime \prime}\right) \uplus \operatorname{DropIds}\left(S^{\prime \prime \prime}\right) \\
& =\quad N o I d s\left(\phi\left(B^{\prime}\right) \uplus\{c \# i\} \uplus G^{\prime \prime \prime}\right) \uplus \operatorname{Drop} I d s\left(S n^{\prime \prime \prime}\right) \quad(\mathbf{a}),(\mathbf{a}) \\
& =\operatorname{NoIds}\left(G^{\prime \prime}\right) \uplus \operatorname{DropIds}\left(S^{\prime \prime}\right)
\end{aligned}
$$

Therefore, we have $\operatorname{NoId}(G) \uplus \operatorname{DropIds}(S n) \longmapsto_{\mathcal{A}}^{*} \operatorname{NoId}\left(G^{\prime}\right) \uplus \operatorname{DropIds}\left(\operatorname{Sn}^{\prime}\right) \longmapsto_{\mathcal{A}}$ $\operatorname{NoIds}\left(G^{\prime \prime}\right) \uplus \operatorname{DropIds}\left(S n^{\prime \prime}\right)$, such that the $k+1$ goal-based derivation step satisfy condition (C2).

- (Drop) $k+1$ step is of the form $\left\langle\{c \# i\} \uplus G^{\prime \prime} \mid S n^{\prime}\right\rangle \stackrel{\delta}{\longmapsto} \mathcal{G}\left\langle\left\{G^{\prime \prime}\left|S n^{\prime}\right\rangle\right.\right.$ such that for some $G^{\prime \prime \prime}$

$$
G^{\prime \prime}=\{c \# i\} \uplus G^{\prime} \text { and } S n^{\prime}=S n^{\prime \prime} \quad\left(\mathbf{a}_{\mathbf{d r o p}}\right)
$$

Hence,

$$
\begin{array}{rll}
\operatorname{NoIds}\left(G^{\prime \prime}\right) \uplus \operatorname{DropIds}\left(\operatorname{Sn}^{\prime \prime}\right) & =\operatorname{NoIds}\left(\{c \# i\} \uplus G^{\prime}\right) \uplus \operatorname{DropIds}\left(\operatorname{Sn}^{\prime}\right) & \text { (a drop) } \\
& =\operatorname{NoIds}\left(G^{\prime}\right) \uplus \operatorname{DropIds}\left(\operatorname{Sn}^{\prime}\right) &
\end{array}
$$


Hence we can conclude that evaluated store of derivation step $k+1$ is equivalent to abstract store of evaluated store of step $k$, therefore satisfying condition (C1).

Considering all forms of $k+1$ derivation steps, (Solve), (Activate) and (Drop) satisfies condition $b f(C 1)$, while (Simplify) and (Propagate) satisfy condition (C2). Hence we can conclude that Theorem 2 holds.

Lemma 1 ( $k$-Concurrency) For any finite $k$ of mutually non-overlapping concurrent derivations,

$$
\begin{aligned}
& \left\langle G_{1} \mid H_{S 1} \cup \cdots \cup H_{S i} \cup \cdots \cup H_{S k} \cup S\right\rangle \stackrel{H_{P 1} \backslash H_{S 1}}{\longmapsto \| \mathcal{G}}\left\langle G_{1}^{\prime} \mid\{\} \cup \cdots \cup H_{S i} \cup \cdots \cup H_{S k} \cup S\right\rangle \\
& \text {.. } \\
& \left\langle G_{i} \mid H_{S 1} \cup \cdots \cup H_{S i} \cup \cdots \cup H_{S k} \cup S\right\rangle \stackrel{H_{P i} \backslash H_{S i}}{\longmapsto}|| \mathcal{G}\left\langle G_{i}^{\prime} \mid H_{S 1} \cup \cdots \cup\{\} \cup \cdots \cup H_{S k} \cup S\right\rangle \\
& \left\langle G_{k} \mid H_{S 1} \cup \cdots \cup H_{S i} \cup \cdots \cup H_{S k} \cup S\right\rangle \stackrel{H_{P k} \backslash H_{S k}}{\longmapsto} \| G_{k}^{\prime}\left|H_{S 1} \cup \cdots \cup H_{S i} \cup \cdots \cup\{\} \cup S\right\rangle \\
& H_{P 1} \subseteq S \cdots H_{P i} \subseteq S \cdots H_{P k} \subseteq S \\
& \delta=H_{P 1} \cup \cdots \cup H_{P i} \cup \cdots \cup H_{P k} \backslash H_{S 1} \cup \cdots \cup H_{S i} \cup \cdots \cup H_{S k} \\
& \begin{aligned}
& \left\langle G_{1} \uplus \cdots \uplus G_{i} \uplus \cdots \uplus G_{k} \uplus G \mid H_{S 1} \cup \cdots \cup H_{S i} \cup \cdots \cup H_{S k} \cup S\right\rangle \\
\stackrel{\delta}{\| \mathcal{G}}^{\prime} & \left\langle G_{1}^{\prime} \uplus \cdots \uplus G_{i}^{\prime} \uplus \cdots \uplus G_{k}^{\prime} \uplus G \mid S\right\rangle
\end{aligned}
\end{aligned}
$$

we can decompose this into $k-1$ applications of the (pair-wise) (Goal Concurrency) derivation step.

Proof

We prove the soundness of $k$-concurrency by showing that $k$ mutually non-overlapping concurrent derivation can be decomposed into $k-1$ applications of (Goal Concurrency) step. We prove by induction on the number of concurrent derivations $k$.

Base case: $k=2$. 2-concurrency immediately corresponds to (Goal Concurrency) rule, hence it is true by definition.

Inductive case: We assume that for $j>2$ and $j<k$, we can decompose $j$ mutually non-overlapping concurrent derivations. into $j-1$ applications of the (Goal Concurrency) step. We now consider $j+1$ mutually non-overlapping concurrent derivations. Because all derivations are non-overlapping, we can compose any two derivations amongst these $j+1$ into a single concurrent step via the (Goal Concurrency) rule. We pick any two concurrent derivations, say the $j^{\text {th }}$ and $(j+1)^{t h}$ (Note that by symmetry, this choice is arbitrary):

$$
\begin{gathered}
\left\langle G_{j} \mid H_{S 1} \cup \cdots \cup H_{S j} \cup H_{S j+1} \cup S\right\rangle \stackrel{H_{P j} \backslash H_{S j}}{\longmapsto \| \mathcal{G}}\left\langle G_{j}^{\prime} \mid H_{S 1} \cup \cdots \cup\{\} \cup H_{S j+1} \cup S\right\rangle \\
\left\langle G_{j+1} \mid H_{S 1} \cup \cdots \cup H_{S j} \cup H_{S j+1} \cup S\right\rangle \stackrel{H_{P j+1} \backslash H_{S j+1}}{\longmapsto}\left\langle G_{j+1}^{\prime} \mid H_{S 1} \cup \cdots \cup H_{S j} \cup\{\} \cup S\right\rangle \\
H_{P j} \subseteq S \quad H_{P j+1} \subseteq S
\end{gathered}
$$

By applying the above two non-overlapping derivations with an instance of the (Goal Concurrency) rule, we have:

$$
\begin{gathered}
\left\langle G_{j^{\prime}} \mid H_{S 1} \cup \cdots \cup H_{S j^{\prime}} \cup S\right\rangle \stackrel{H_{P j^{\prime}} \backslash H_{S j^{\prime}}}{\longmapsto}\left\langle G^{\prime}\left|G_{j^{\prime}}\right| H_{S 1} \cup \cdots \cup\{\} \cup S\right\rangle \\
\text { where } \quad G_{j^{\prime}}=G_{j} \uplus G_{j+1} \quad G_{j^{\prime}}^{\prime}=G_{j}^{\prime} \uplus G_{j+1}^{\prime} \\
H_{S j^{\prime}}=H_{S j} \cup H_{S j+1} \quad H_{P j^{\prime}}=H_{P j} \cup H_{P j+1}
\end{gathered}
$$


Hence we have reduced $j+1$ non-overlapping concurrent derivations into $j$ non-overlapping concurrent derivations by combining via the (Goal Concurrency) derivation step.

$$
\begin{gathered}
\left\langle G_{1} \mid H_{S 1} \cup \cdots \cup H_{S j^{\prime}} \cup S\right\rangle \stackrel{H_{P 1} \backslash H_{S 1}}{\longmapsto} \| G_{1}^{\prime}\left|\{\} \cup \cdots \cup H_{S j^{\prime}} \cup S\right\rangle \\
\quad . . \\
\left\langle G_{j^{\prime}} \mid H_{S 1} \cup \cdots \cup H_{S j^{\prime}} \cup S\right\rangle \stackrel{H_{P j^{\prime}} \backslash H_{S j^{\prime}}}{\longmapsto} \| G_{j^{\prime}}^{\prime}\left|H_{S 1} \cup \cdots \cup\{\} \cup S\right\rangle \\
H_{P 1} \subseteq S \cdots H_{P j^{\prime}} \subseteq S \\
\delta=H_{P 1} \cup \cdots \cup H_{P j^{\prime}} \backslash H_{S 1} \cup \cdots \cup H_{S j^{\prime}} \\
\qquad G_{1} \uplus \cdots \uplus G_{j^{\prime}} \uplus G\left|H_{S 1} \cup \cdots \cup H_{S j^{\prime}} \cup S\right\rangle \\
\qquad \| \mathcal{G} \quad\left\langle G_{1}^{\prime} \uplus \cdots \uplus G_{j^{\prime}}^{\prime} \uplus G \mid S\right\rangle
\end{gathered}
$$

Hence, by our original assumption, the above is decomposable into $j-1$ applications of the (Goal Concurrency) step. This implies that $j+1$ concurrent derivations are decomposable into $j$ (Goal Concurrency) step.

Lemma 2 (Monotonicity of Goals in Goal-based Semantics) For any goals $G, G^{\prime}$ and $G^{\prime \prime}$ and CHR store $S n$ and $S n^{\prime}$, if $\langle G \mid S n\rangle \longmapsto_{\mathcal{G}}^{*}\left\langle G^{\prime} \mid S n^{\prime}\right\rangle$ then $\left\langle G \uplus G^{\prime \prime}\right|$ $S n\rangle \longmapsto_{\mathcal{G}}^{*}\left\langle G^{\prime} \uplus G^{\prime \prime} \mid S n^{\prime}\right\rangle$

\section{Proof}

We need to prove that for any finite $k$, if $\langle G \mid S n\rangle \hookrightarrow_{\mathcal{G}}^{k}\left\langle G^{\prime} \mid S n^{\prime}\right\rangle$ we can always extend the goals with any $G^{\prime \prime}$ such that $\left\langle G \uplus G^{\prime \prime} \mid S n\right\rangle \longmapsto_{\mathcal{G}}^{k}\left\langle G^{\prime} \uplus G^{\prime \prime} \mid S n^{\prime}\right\rangle$.

We prove this by induction on the number of derivation steps $k$, showing that for any finite $i \leq k$, goals are monotonic.

Base case: We consider $\langle G \mid S n\rangle \longmapsto_{\mathcal{G}}^{0}\left\langle G^{\prime} \mid S n^{\prime}\right\rangle$. By definition of $\longmapsto_{\mathcal{G}}^{0}$, we have $G=G^{\prime}$ and $S n=S n^{\prime}$. Hence we immediately have $\left\langle G \uplus G^{\prime \prime} \mid S n\right\rangle \longmapsto_{\mathcal{G}}^{0}\left\langle G^{\prime} \uplus G^{\prime \prime}\right|$ $\left.S n^{\prime}\right\rangle$

Inductive case: We assume that the lemma is true for some finite $i>0$, hence $\langle G \mid S n\rangle \longmapsto_{\mathcal{G}}^{i}\left\langle G^{\prime} \mid S n^{\prime}\right\rangle$ is monotonic with respect to the goals.

We now prove that by extending these $i$ derivations with another step, we still preserve monotonicity of the goals. Namely, if $\langle G \mid S n\rangle \longmapsto_{\mathcal{G}}^{i}\left\langle\{g\} \uplus G_{i} \mid S n_{i}\right\rangle \stackrel{\delta}{\longmapsto} \mathcal{G}$ $\left\langle G_{i+1} \mid S n_{i+1}\right\rangle$ then $\left\langle G \uplus G^{\prime \prime} \mid S n\right\rangle \longmapsto_{\mathcal{G}}^{i}\left\langle G_{i} \uplus G^{\prime \prime} \mid S n_{i}\right\rangle \stackrel{\delta}{\longmapsto} \mathcal{G}\left\langle G_{i+1} \uplus G^{\prime \prime} \mid S n_{i+1}\right\rangle$ We prove this by considering all possible form of derivation step, step $i+1^{\text {th }}$ can take:

- (Solve) Consider $i+1^{\text {th }}$ derivation step of the form $\left\langle\{e\} \uplus G_{i} \mid S n_{i}\right\rangle \longmapsto_{\mathcal{G}}\langle W \uplus G|$ $\left.\{e\} \cup S n_{i}\right\rangle$ for some equation $e$ and $W=\operatorname{WakeUp}\left(e, S n_{i}\right)$.

By definition, the (Solve) step only make reference to $e$ and $S n_{i}$, hence we can extend $G_{i}$ with any $G^{\prime \prime}$ without affecting the derivation step, i.e.

$$
\left\langle\{e\} \uplus G_{i} \uplus G^{\prime \prime} \mid S n_{i}\right\rangle \longmapsto \mathcal{G}\left\langle W \uplus G_{i} \uplus G^{\prime \prime} \mid\{e\} \cup S n_{i}\right\rangle
$$

Hence, given our assumption that the first $i$ derivations are monotonic with respect to the goals, extending with a $i+1^{\text {th }}$ (Solve) step preserves monotonicity of the goals. 
- (Activate) Consider $i+1^{\text {th }}$ derivation step of the form $\left\langle\{c\} \uplus G_{i} \mid S n_{i}\right\rangle \longmapsto \mathcal{G}\langle\{c \# j\} \uplus$ $G_{i}\left|\{c \# j\} \cup S n_{i}\right\rangle$ for some CHR constraint $c$, goals $G_{i}$ and store $S n_{i}$.

By definition, the (Activate) step only make reference to goal $c$, hence we can extend $G_{i}$ with any $G^{\prime \prime}$ without affecting the derivation step, i.e.

$$
\left\langle\{c\} \uplus G_{i} \uplus G^{\prime \prime} \mid S n_{i}\right\rangle \longmapsto_{\mathcal{G}}\left\langle\{c \# j\} \uplus G_{i} \uplus G^{\prime \prime} \mid\{c \# j\} \cup S n_{i}\right\rangle
$$

Hence, given our assumption that the first $i$ derivations are monotonic with respect to the goals, extending with a $i+1^{\text {th }}$ (Activate) step preserves monotonicity of the goals.

- (Simplify) Consider $i+1^{\text {th }}$ derivation step of the form $\left\langle\{c \# j\} \uplus G_{i}\right|\{c \# j\} \uplus H_{S} \cup$

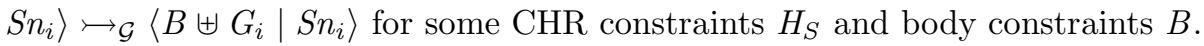

By definition, the (Simplify) step only make reference to goal $c \# j$, and $H_{S}$ of the store, hence we can extend $G_{i}$ with any $G^{\prime \prime}$ without affecting the derivation step, i.e.

$$
\left\langle\{c \# j\} \uplus G_{i} \uplus G^{\prime \prime} \mid\{c \# j\} \cup H_{S} \cup S n_{i}\right\rangle \longmapsto_{\mathcal{G}}\left\langle B \uplus G_{i} \uplus G^{\prime \prime} \mid S n_{i}\right\rangle
$$

Hence, given our assumption that the first $i$ derivations are monotonic with respect to the goals, extending with a $i+1^{\text {th }}$ (Simplify) step preserves monotonicity of the goals.

- (Propagate) Consider $i+1^{\text {th }}$ derivation step of the form $\left\langle\{c \# j\} \uplus G_{i} \mid H_{S} \cup S n_{i}\right\rangle \longmapsto \mathcal{G}$ $\left\langle B \uplus\{c \# j\} \uplus G_{i} \mid S n_{i}\right\rangle$ for some CHR constraints $H_{S}$ and body constraints $B$.

By definition, the (Propagate) step only make reference to goal $c \# j$, and $H_{S}$ of the store, hence we can extend $G_{i}$ with any $G^{\prime \prime}$ without affecting the derivation step, i.e.

$$
\left\langle\{c \# j\} \uplus G_{i} \uplus G^{\prime \prime} \mid H_{S} \cup S n_{i}\right\rangle \longmapsto_{\mathcal{G}}\left\langle B \uplus\{c \# j\} \uplus G_{i} \uplus G^{\prime \prime} \mid S n_{i}\right\rangle
$$

Hence, given our assumption that the first $i$ derivations are monotonic with respect to the goals, extending with a $i+1^{t h}$ (Propagate) step preserves monotonicity of the goals.

- (Drop) Consider $i+1^{\text {th }}$ derivation step of the form $\left\langle\{c \# j\} \uplus G_{i} \mid S n_{i}\right\rangle \longmapsto \mathcal{G}\left\langle G_{i} \mid S n_{i}\right\rangle$ for some numbered constraint $c \# j$.

By definition, the (Drop) step only make reference to goal $c \# j$, while its premise depend on $S n_{i}$, hence we can extend goals $G_{i}$ with any $G^{\prime \prime}$ without affecting the derivation step, i.e.

$$
\left\langle\{c \# j\} \uplus G_{i} \uplus G^{\prime \prime} \mid S n_{i}\right\rangle \longmapsto_{\mathcal{G}}\left\langle G_{i} \uplus G^{\prime \prime} \mid S n_{i}\right\rangle
$$

Hence, given our assumption that the first $i$ derivations are monotonic with respect to the goals, extending with a $i+1^{\text {th }}$ (Drop) step preserves monotonicity of the goals.

Hence, with our assumption of monotonicity of goals for $i$ steps, the goals are still monotonic for $i+1$ steps regardless of the form of the $i+1^{\text {th }}$ derivation step.

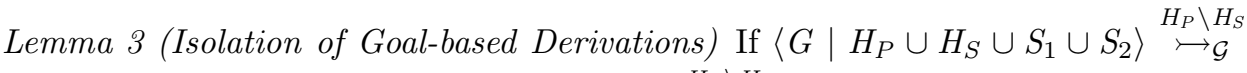
$\left\langle G^{\prime} \mid H_{P} \cup S_{1}^{\prime} \cup S_{2}\right\rangle$ then $\left\langle G \mid H_{P} \cup H_{S} \cup S_{1}\right\rangle \stackrel{H_{P} \backslash H_{S}}{\longmapsto \mathcal{G}}\left\langle G^{\prime} \mid H_{P} \cup S_{1}^{\prime}\right\rangle$ 
Proof

We need to show that for any goal-based derivation, we can omit any constraint of the store which is not a side-effect of the derivation. To prove this, we consider all possible forms of goal-based derivations:

- (Solve) Consider derivation of the form

$$
\left\langle\{e\} \uplus G \mid W \cup\{\} \cup S_{1} \cup S_{2}\right\rangle \stackrel{W \backslash\{\}}{\longmapsto \mathcal{G}}\left\langle W \uplus G \mid W \cup\{\} \cup\{e\} \cup S_{1} \cup S_{2}\right\rangle
$$

Since wake up side-effect is captured in $W$, we can drop $S_{2}$ without affecting the derivation. Hence we also have:

$$
\left\langle\{e\} \uplus G \mid W \cup\{\} \cup S_{1}\right\rangle \stackrel{W \backslash\{\}}{\longmapsto \mathcal{G}}\left\langle W \uplus G \mid W \cup\{\} \cup\{e\} \cup S_{1}\right\rangle
$$

- (Activate) Consider derivation of the form

$$
\left\langle\{c\} \uplus G \mid\{\} \cup\{\} \cup S_{1} \cup S_{2}\right\rangle \stackrel{\{\} \backslash\{\}}{\longmapsto \mathcal{G}}\left\langle\{c \# i\} \uplus G \mid\{\} \cup\{\} \cup\{c \# i\} \cup S_{1} \cup S_{2}\right\rangle
$$

Since (Activate) simply introduces a new constraint $c \# i$ into the store, we can drop $S_{2}$ without affecting the derivation. Hence we also have:

$$
\left\langle\{c\} \uplus G \mid\{\} \cup\{\} \cup S_{1}\right\rangle \stackrel{\{\} \backslash\{\}}{\longmapsto \mathcal{G}}\left\langle\{c \# i\} \uplus G \mid\{\} \cup\{\} \cup\{c \# i\} \cup S_{1}\right\rangle
$$

- (Simplify) Consider derivation of the form

$$
\left\langle\{c \# i\} \uplus G \mid H_{P} \cup H_{S} \cup S_{1} \cup S_{2}\right\rangle \stackrel{H_{P} \backslash H_{S}}{\longmapsto \mathcal{G}}\left\langle B \uplus G \mid H_{P} \cup S_{1} \cup S_{2}\right\rangle
$$

Since $S_{2}$ is not part of the side-effects of this derivation, we can drop $S_{2}$ without affecting the derivation. Hence we also have:

$$
\left\langle\{c \# i\} \uplus G \mid H_{P} \cup H_{S} \cup S_{1}\right\rangle \stackrel{H_{P} \backslash H_{S}}{\longmapsto \mathcal{G}}\left\langle B \uplus G \mid H_{P} \cup S_{1}\right\rangle
$$

- (Propagate) Consider derivation of the form

$$
\left\langle\{c \# i\} \uplus G \mid H_{P} \cup H_{S} \cup S_{1} \cup S_{2}\right\rangle \stackrel{H_{P} \backslash H_{S}}{\longmapsto \mathcal{G}}\left\langle B \uplus\{c \# i\} \uplus G \mid H_{P} \cup S_{1} \cup S_{2}\right\rangle
$$

Since $S_{2}$ is not part of the side-effects of this derivation, we can drop $S_{2}$ without affecting the derivation. Hence we also have:

$$
\left\langle\{c \# i\} \uplus G \mid H_{P} \cup H_{S} \cup S_{1}\right\rangle \stackrel{H_{P} \backslash H_{S}}{\longmapsto \mathcal{G}}\left\langle B \uplus\{c \# i\} \uplus G \mid H_{P} \cup S_{1}\right\rangle
$$

- (Drop) Consider derivation of the form

$$
\left\langle\{c \# i\} \uplus G \mid\{\} \cup\{\} \cup S_{1} \cup S_{2}\right\rangle \stackrel{\{\} \backslash\{\}}{\longmapsto \mathcal{G}}\left\langle G \mid\{\} \cup\{\} \cup S_{1} \cup S_{2}\right\rangle
$$

(Drop) simply removes the goal $c \# i$ when no instances of (Simplify) or (Propagate) can apply on it. Note that its premise references to the entire store, so removing $S_{2}$ may seems unsafe. But since removing constraints from the store will not cause $c \# i$ to be applicable to any instances of (Simplify) or (Propagate), hence we also have:

$$
\left\langle\{c\} \uplus G \mid\{\} \cup\{\} \cup S_{1}\right\rangle \stackrel{\{\} \backslash\{\}}{\longmapsto \mathcal{G}}\left\langle G \mid\{\} \cup\{\} \cup S_{1}\right\rangle
$$


Lemma 4 (Isolation of Transitive Goal-based Derivations) If $\langle G| H_{P} \cup H_{S} \cup S_{1} \cup$ $\left.S_{2}\right\rangle \longmapsto_{\mathcal{G}}^{*}\left\langle G^{\prime} \mid H_{P} \cup S_{1}^{\prime} \cup S_{2}\right\rangle$ with side-effects $\delta=H_{P} \backslash H_{S}$, then $\left\langle G \mid H_{P} \cup H_{S} \cup S_{1}\right\rangle \longmapsto_{\mathcal{G}}^{*}$ $\left\langle G^{\prime} \mid H_{P} \cup S_{1}^{\prime}\right\rangle$

Proof

We need to prove that for all $k,\left\langle G \mid H_{P} \cup H_{S} \cup S_{1} \cup S_{2}\right\rangle \stackrel{k}{\longmapsto_{\mathcal{G}}}\left\langle G^{\prime} \mid H_{P} \cup S_{1}^{\prime} \cup S_{2}\right\rangle$ with side-effects $\delta=H_{P} \backslash H_{S}$ we can always safely omit affected potions of the store from the derivation. We prove by induction on $i \leq k$.

Base case: $i=1$. Consider, $\left\langle G \mid H_{P} \cup H_{S} \cup S_{1} \cup S_{2}\right\rangle \hookrightarrow_{\mathcal{G}}^{1}\left\langle G^{\prime} \mid H_{P} \cup S_{1}^{\prime} \cup S_{2}\right\rangle$. This corresponds to the premise in Lemma 3 , hence we can safely omit $S_{2}$ from the derivation.

Inductive case: $i>1$. we assume that for any $\left\langle G \mid H_{P i} \cup H_{S i} \cup S_{1 i} \cup S_{2 i}\right\rangle \longmapsto_{\mathcal{G}}^{i}$ $\left\langle G^{\prime} \mid H_{P i} \cup S_{1 i}^{\prime} \cup S_{2 i}\right\rangle$ with side-effects $\delta_{i}=H_{P i} \backslash H_{S i}$, we can safely omit $S_{2 i}$ from the derivation. Let's consider a $j=i+1$ derivation step from here, which contains side-effects $\delta_{j}=H_{P j} \backslash H_{S j}$ non-overlapping with $\delta_{i}$. Hence $H_{P j}$ and $H_{S j}$ must be in $S_{2 i}$ (i.e. $S_{2 i}=H_{P j} \cup H_{S j} \cup S_{1 j} \cup S_{2 j}$ ).

$$
\begin{aligned}
& \left\langle G \mid H_{P i} \cup H_{S i} \cup S_{1 i} \cup H_{P j} \cup H_{S j} \cup S_{1 j} \cup S_{2 j}\right\rangle \\
\longmapsto_{\mathcal{G}}^{i} & \left\langle G^{\prime} \mid H_{P} \cup S_{1 i}^{\prime} \cup H_{P j} \cup H_{S j} \cup S_{1 j} \cup S_{2 j}\right\rangle \\
\delta_{j} & \left\langle G^{\prime \prime} \mid H_{P} \cup S_{1 i}^{\prime} \cup H_{P j} \cup S_{1 j}^{\prime} \cup S_{2 j}\right\rangle
\end{aligned}
$$

Hence consider the following substitutions:

$$
\begin{array}{ll}
H_{P}=H_{P i} \cup H_{P j} & H_{S}=H_{S i} \cup H_{S j} \\
S_{1}=S_{1 i} \cup S_{1 j} & S_{1}^{\prime}=S_{1 i}^{\prime} \cup S_{1 j}^{\prime} \\
\delta=H_{P} \backslash H_{S} &
\end{array}
$$

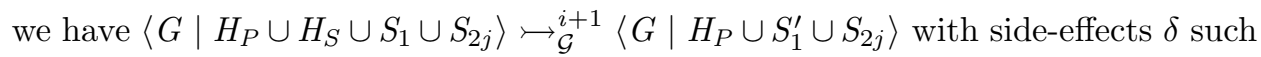
that no constraints in $S_{2 j}$ is in $\delta$. Hence we can safely omit $S_{2 j}$ from the derivation and we have isolation for $i+1$ derivations as well.

Lemma可 (Sequential Reachability of Concurrent Derivation Steps) For any sequentially reachable CHR state $\sigma$, CHR state $\sigma^{\prime}$ and rewriting side-effects $\delta$ if $\sigma \stackrel{\delta}{\| \mathcal{G}} \sigma^{\prime}$ then $\sigma^{\prime}$ is sequentially reachable, $\sigma \longmapsto_{\mathcal{G}}^{*} \sigma^{\prime}$ with side-effects $\delta$.

Proof

From the $k$-concurrency Lemma (Lemma 1) we showed that any finite $k$ mutually non-overlapping concurrent goal-based derivations can be replicated by nested application of the (Goal Concurrency) step. Hence, to prove sequential reachability of concurrent derivations, we only need to consider the derivation steps (Lift) and (Goal Concurrency) which sufficiently covers the concurrent behaviour of any $k$ concurrent derivations.

We prove by structural induction of the concurrent goal-based semantics derivation steps (Lift) and (Goal Concurrency).

- (Lift) is the base case. Application of (Lift) simply lifts a goal-based derivation $\sigma \stackrel{\delta}{\longmapsto} \mathcal{G} \sigma^{\prime}$ into a concurrent goal-based derivation $\sigma \stackrel{\delta}{\longmapsto} \| \mathcal{G} \sigma^{\prime}$. Thus states $\sigma^{\prime}$ derived 
from the (Lift) step is immediately sequentially reachable since $\sigma \stackrel{\delta}{\longmapsto} \mathcal{G} \sigma^{\prime}$ implies $\sigma \longmapsto \stackrel{*}{\mathcal{G}} \sigma^{\prime}$.

- (Goal Concurrency)

$$
\begin{aligned}
& \text { (D1) }\left\langle G_{1} \mid H_{S 1} \cup H_{S 2} \cup S\right\rangle \stackrel{\delta_{1}}{\|_{\mathcal{G}}}\left\langle G_{1}^{\prime} \mid\{\} \cup H_{S 2} \cup S\right\rangle \\
& \text { (D2) }\left\langle G_{2} \mid H_{S 1} \cup H_{S 2} \cup S\right\rangle \stackrel{\delta_{2}}{\longmapsto}\left\langle\mathcal{G}\left\langle G_{2}^{\prime} \mid H_{S 1} \cup\{\} \cup S\right\rangle\right. \\
& \delta_{1}=H_{P 1} \backslash H_{S 1} \quad \delta_{2}=H_{P 2} \backslash H_{S 2} \\
& H_{P 1} \subseteq S \quad H_{P 2} \subseteq S \quad \delta=H_{P 1} \cup H_{P 2} \backslash H_{S 1} \cup H_{S 2} \\
& \begin{aligned}
& \left\langle G_{1} \uplus G_{2} \uplus G \mid H_{S 1} \cup H_{S 2} \cup S\right\rangle \\
(\mathbf{C}) \quad \stackrel{\delta}{\longmapsto} \| \mathcal{G} & \left\langle G_{1}^{\prime} \uplus G_{2}^{\prime} \uplus G \mid S\right\rangle
\end{aligned}
\end{aligned}
$$

we assume that (D1) and (D2) are sequentially reachable. This means that we have the following:

$$
\begin{aligned}
& \left\langle G_{1} \mid H_{S 1} \cup H_{S 2} \cup S\right\rangle \longmapsto_{\mathcal{G}}^{*}\left\langle G_{1}^{\prime} \mid\{\} \cup H_{S 2} \cup S\right\rangle \\
& \text { with side-effects } \delta_{1}=H_{P 1} \backslash H_{S 1} \text { such that } H_{P 1} \subseteq S \quad \text { (aD1) } \\
& \left\langle G_{2} \mid H_{S 1} \cup H_{S 2} \cup S\right\rangle \longmapsto_{\mathcal{G}}^{*}\left\langle G_{2}^{\prime} \mid H_{S 1} \cup\{\} \cup S\right\rangle \\
& \text { with side-effects } \delta_{2}=H_{P 2} \backslash H_{S 2} \text { such that } H_{P 2} \subseteq S \quad \text { (a.D2) }
\end{aligned}
$$

Since both derivations are by definition non-overlapping in side-effects, we can show that (C) is sequentially reachable, using monotonicity of goals (Lemma 2) and isolation of derivations (Lemma 3):

$$
\begin{aligned}
& \left\langle G_{1} \uplus G_{2} \uplus G \mid H_{S 1} \cup H_{S 2} \cup S\right\rangle \\
& \left.\longmapsto_{\mathcal{G}}^{*} \quad\left\langle G_{1}^{\prime} \uplus G_{2} \uplus G \mid H_{S 2} \cup S\right\rangle \quad \text { (Lemma2, a a } 1\right) \\
& \longmapsto_{\mathcal{G}}^{*}\left\langle G_{1}^{\prime} \uplus G_{2}^{\prime} \uplus G \mid S\right\rangle \quad \text { (Lemma2, Lemma[4, a a }
\end{aligned}
$$

Hence, the above sequential goal-based derivation shows that (Goal Concurrency) derivation step is sequentially reachable with side-effect $\delta$.

Theorem 3 (Sequential Reachability of Concurrent Derivations) For any initial CHR state $\sigma$, CHR state $\sigma^{\prime}$ and CHR Program $\mathcal{P}$, if $\sigma \longmapsto_{\| \mathcal{G}}^{*} \sigma^{\prime}$ then $\sigma \longmapsto_{\mathcal{G}}^{*} \sigma^{\prime}$.

Proof

We prove that for all finite $k$ number of concurrent derivation steps $\sigma \longmapsto \| \mathcal{G}^{k} \sigma^{\prime}$, we can find a corresponding sequential derivation sequence $\sigma \longmapsto_{\mathcal{G}}^{*} \sigma^{\prime}$.

Base case: $k=1$. We consider $\sigma \longmapsto \|_{\| \mathcal{G}}^{1} \sigma^{\prime}$. From Lemma 5, we can conclude that we have $\sigma \longmapsto{ }_{\mathcal{G}}^{*} \sigma^{\prime}$ as well.

Inductive case: $k>1$. We consider $\sigma \longmapsto_{\| \mathcal{G}}^{k} \sigma^{\prime}$, assuming that it is sequentially reachable, hence we also have $\sigma \longmapsto{ }_{\mathcal{G}}^{*} \sigma^{\prime}$. We consider extending this derivation with the $k+1^{\text {th }}$ step $\sigma^{\prime} \longmapsto_{\| \mathcal{G}} \sigma^{\prime \prime}$. By Lemma 5, we can conclude that the $k+1^{\text {th }}$ concurrent derivation is sequential reachable, hence $\sigma^{\prime} \longmapsto_{\mathcal{G}}^{*} \sigma^{\prime \prime}$. Hence we have $\sigma \longmapsto_{\mathcal{G}}^{*} \sigma^{\prime} \longmapsto{ }_{\mathcal{G}}^{*} \sigma^{\prime \prime}$ showing that $\sigma{ }_{\| \mathcal{G}}^{k+1} \sigma^{\prime \prime}$ is sequentially reachable. 


\subsubsection{Correspondence of Termination}

Lemma 6 (Rule instances in reachable states are always active) For any reachable CHR state $\langle G \mid S n\rangle$, any rule head instance $H \subseteq S n$ must be active. i.e. $\exists c \# i \in H$ such that $c \# i \in G$.

Proof

We will prove this for the sequential goal-based semantics. Since Theorem 3 states all concurrent derivation is sequentially reachable, this Lemma immediately applies to the concurrent goal-based semantics as well.

We prove that for all finite $k$ derivations from any initial CHR state $\langle G \mid\{\}\rangle$, i.e. $\langle G \mid\{\}\rangle \longmapsto_{\mathcal{G}}^{k}\left\langle G^{\prime} \mid S n^{\prime}\right\rangle$, all rule head instances $H \subseteq S n^{\prime}$ has at least one $c \# i \in H$ such that $c \# i \in G$. We prove by induction on $i<k$ that states reachable by $i$ derivations from an initial stage have the above property.

Base case: $i=0$. Hence $\langle G \mid\{\}\rangle \longmapsto_{\mathcal{G}}^{0}\left\langle G^{\prime} \mid S n^{\prime}\right\rangle$. By definition, $G=G^{\prime}$ and $S n^{\prime}=\{\}$. Since $S n^{\prime}$ is empty, the base case immediately satisfies the Lemma.

Inductive case: $i>0$. We assume that for any $\langle G \mid\{\}\rangle \longmapsto_{\mathcal{G}}^{i}\left\langle G^{\prime} \mid S n^{\prime}\right\rangle$, all rule head instances $H \subseteq S n^{\prime}$ is active, hence have at least one $c \# i \in H$ such that $c \# i \in G^{\prime}$. We extend this derivation with an $i+1^{\text {th }}$ step, hence $\langle G \mid\{\}\rangle \longmapsto_{\mathcal{G}}^{i}\left\langle G^{\prime}\right|$ $\left.S n^{\prime}\right\rangle \stackrel{\delta}{\longmapsto} \mathcal{G}\left\langle G^{\prime \prime} \mid S n^{\prime \prime}\right\rangle$. We now prove that all rule head instances in $S n^{\prime \prime}$ are active. We consider all possible forms of this $i+1^{\text {th }}$ derivation step. We omit side-effects.

- (Solve) $i+1$ derivation step is of the form $\left\langle\{e\} \uplus G^{\prime \prime \prime} \mid S n^{\prime}\right\rangle \longmapsto_{\mathcal{G}}\left\langle W \uplus G^{\prime \prime \prime} \mid\{e\} \cup S n^{\prime}\right\rangle$ for some goals $G^{\prime \prime \prime}$ and $W=$ WakeUp $\left(e, S n^{\prime}\right)$. Our assumption provides that all rule head instances in $S n^{\prime}$ are active. Introducing $e$ into the store will possibly introduce new rule head instances. This is because for some CHR rule $\left(r @ H_{P} \backslash H_{S} \Longleftrightarrow t_{g} \mid\right.$ $B) \in \mathcal{P}$ since we may have a new $\phi$ such that $\operatorname{Eqs}\left(\{e\} \cup S n^{\prime}\right) \models \phi \wedge t_{g}$ and $\phi\left(H_{P} \cup H_{S}\right) \in S n^{\prime}$. This means that there is at least one $c \# i$ in $\phi\left(H_{P} \cup H_{S}\right)$ which is further grounded by $e$. Thankfully, by definition of $W=\operatorname{WakeUp}\left(e, S n^{\prime}\right)$, we have $c \# i \in W$. Hence new rule head instances will become active because of introduction of $W$ to the goals.

- (Activate) $i+1$ derivation step is of the form $\left\langle\{c\} \uplus G^{\prime \prime \prime} \mid S n^{\prime}\right\rangle \mapsto_{\mathcal{G}}\left\langle\{c \# i\} \uplus G^{\prime \prime \prime}\right|$ $\left.\{c \# i\} \cup S n^{\prime}\right\rangle$. Our assumption provides that all rule head instances in $S n^{\prime}$ are active. By adding $c \# i$ to the store, we can possibly introduce new rule head instances $\{c \# i\} \cup H$ such that $H \in S n^{\prime}$. Since $c \# i$ is also retained as a goal, such new rule head instances are active as well.

- (Simplify) $i+1$ derivation step is of the form $\left\langle\{c \# i\} \uplus G^{\prime \prime \prime} \mid\{c \# i\} \cup H_{S} \cup S n^{\prime}\right\rangle \longmapsto \mathcal{G}$ $\left\langle B \uplus G^{\prime \prime \prime} \mid S n^{\prime}\right\rangle$. Our assumption provides that all rule head instances in $S n^{\prime}$ are active. $c \# i$ has applied a rule instance, removing $c \# i$ and some $H_{S}$ from the store. Since $c \# i$ is no longer in the store, we can safely remove $c \# i$ from the goals. Removing $H_{S}$ from the store will only (possibly) remove other rule head instance from the store. Hence rule head instances in $S n^{\prime}$ still remain active.

- (Propagate) $i+1$ derivation step is of the form $\left\langle\{c \# i\} \uplus G^{\prime \prime \prime} \mid\{c \# i\} \cup H_{S} \cup S n^{\prime}\right\rangle \longmapsto_{\mathcal{G}}$ $\left\langle B \uplus\{c \# i\} \uplus G^{\prime \prime \prime} \mid\{c \# i\} \cup S n^{\prime}\right\rangle$. Our assumption provides that all rule head instances in $S n^{\prime}$ are active. $c \# i$ has applied a rule instance, removing some $H_{S}$ from the store. Since $c \# i$ is still in the store, we cannot safely remove $c \# i$ from the goals, thus 
it is retained. Removing $H_{S}$ from the store will only (possibly) remove other rule head instance from the store. Hence rule head instances in $S n^{\prime}$, including those that contains $c \# i$, still remain active.

- (Drop) $i+1$ derivation step is of the form $\left\langle\{c \# i\} \uplus G^{\prime \prime \prime} \mid S n^{\prime}\right\rangle \hookrightarrow_{\mathcal{G}}\left\langle G^{\prime \prime \prime} \mid S n^{\prime}\right\rangle$. Our assumption provides that all rule head instances in $S n^{\prime}$ are active. Premise of the (Drop) step demands that no (Simplify) and (Propagate) steps apply on $c \# i$. This means that $c \# i$ is not part of any rule head instances in $S n^{\prime}$. Hence we can safely remove $c \# i$ from the goals without risking to deactivate any rule instances.

Hence (Solve) and (Activate) guarantees that new rule head instances become active, (Drop) safely removes a goal without deactivating any rule head instances and (Simplify) and (Propagate) only removes constraint from the store. In all cases, existing rule head instances remain active while new rule head instances become active, thus we have proved the lemma.

Theorem 4 (Correspondence of Termination) For any initial CHR state $\langle G,\{\}\rangle$, final CHR state $\langle\{\}, S n\rangle$ and terminating CHR program $\mathcal{P}$,

if $\langle G \mid\{\}\rangle \mapsto_{\| \mathcal{G}}^{*}\langle\{\} \mid S n\rangle$ then $G r_{\mathcal{A}}^{*} \operatorname{DropIds}(S n)$ and $\operatorname{Final}_{\mathcal{A}}(\operatorname{DropIds}(S n))$

Proof

We prove that for any concurrent derivation $\langle G \mid\{\}\rangle \longmapsto_{\| \mathcal{G}}^{*}\langle\{\} \mid S n\rangle$, we have a corresponding abstract derivation $G r_{\mathcal{A}}^{*} \operatorname{DropIds}(S n)$. Theorem 3 states that we can replicate the above concurrent derivation, with a sequential derivation. Hence we have $\langle G \mid\{\}\rangle \hookrightarrow_{\mathcal{G}}^{*}\langle\{\} \mid S n\rangle$. By instantiating Theorem 2. we immediately have

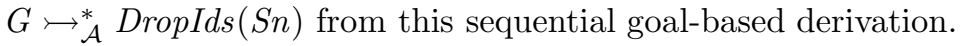

Next we show that $\operatorname{DropIds}(S n)$ is a final store $\left(\operatorname{Final}_{\mathcal{A}}\left(\operatorname{DropIds}_{(S n)))}\right)\right.$ with respect to some CHR program $\mathcal{P}$. We prove by contradiction: Suppose $\operatorname{DropIds}(S n)$ is not a final store, hence $\langle\{\} \mid S n\rangle$ has at least one rule head instance $H$ of $\mathcal{P}$ in $S n$ which is not active, since the goals are empty. However, this contradicts with Lemma 6. which states that all reachable states have only active rule instances. Since $\langle\{\} \mid S n\rangle$ is sequentially reachable, it must be the case that $S n$ has no rule head instances of $\mathcal{P}$. Therefore $\operatorname{DropIds}(S n)$ must be a final store. 